\title{
Using Power Series Expansions of Moduli to Interpolate between Release Curves from Dynamic Tests: Technique and Application
}

\author{
M.D. Furnish \\ Division 1534 Sandia National Laboratories \\ Albuquerque, New Mexico 87185
}

\begin{abstract}
Recently an appreciable number of continuous release profiles have been measured from dynamic experiments with geological materials. For each material an empirical generalization of the available release curves may be constructed to allow easy application of the experimental data to problems in much the same way as a linear shock velocity - particle velocity fit allows easy application of Hugoniot data. This generalization is made in two steps. The first is to compute the Eulerian axial modulus at the Hugoniot pressure and its first three pressure derivatives along the release for each test. This corresponds to a partial Taylor series of the axial modulus, which integrates to give a very close match to the original release. An alternative formulation, which takes volume as the independent variable, fails becavse that Taylor series does not converge with the rapidity needed for these calculations. The second step is to plot eack: of these quantities against the Hugoniot pressure for the suite of tests, and fit these data. A release from an arbitrary pressure within the general range of the experimental data may be computed by using the interpolated modu' $\lrcorner s$ and its interpolated derivatives. This generalization, which allows volume to be computed as a function of pressure, reproduces the experimental curves fairly well. We present the results of applying this technique to release data for Mini Jade 2 grout, and briefly compare these results with those from several Nevada Test Site tuffs, saturated and dry Indiana Limestone, and aluminum. Finally, we use the generalized Mini Jade 2 data to solve a sample problem, that of estimating the error produced by making the "release = Hugoniot" assumption in the analysis of ground motion gauges in an underground test.
\end{abstract}




\section{Contents}

1. Introduction . . . . . . . . . . . . . . . . . . . . . . 7

2. Developing a prescription for interpolating release data between tests 9

2.1 Summary of Experimental Technique . . . . . . . . . . . . . 9

2.2 Expressing the release curves as Eulerian moduli and pressure derivatives at. the Hugoniot condition . . . . . . . . . . . . . . . . . . 12

2.3 Attempting to express the release curves in terms of volume derivatives of pressure . . . . . . . . . . . . . . . . . . . . . . . 14

2.4 Linking the modulus data together into the desired algorithm . . . . . 16

3. Empirical EOS summaries for specific materials . . . . . . . . 19

3.1 Mini Jade 2 Grout . . . . . . . . . . . . . . . . . . . 19

3.2 Other materials $\quad . \quad$. . . . . . . . . . . . . . . . . . . . . . 25

4. Application of interpolatable expression of releases to a practical problem . . . . . . . . . . . . . . . . . . . . . . . 29

\section{Tables}

2.1 Equation of State Properties . . . . . . . . . . . . . . . . 12

3.1 Coefficients $B_{i}$ for MJ-2 using a smoothed Hugoniot . . . . . . . . 20

3.2 Equations for fits of axial modulus and pressure derivatives versus Hugoniot pressure for MJ-2 . . . . . . . . . . . . . . . . . . . 22

4.1 Summary of analysis of system with shock propagating from MJ-2 into aluminum or magnesium . . . . . . . . . . . . . . . . . 35

4.2 Summary of analysis of system with shock propagating from MJ-2 into PMMA 36 


\section{Figures}

2.1 Experimental Configuration . . . . . . . . . . . . . . . . 10

2.2 Wave Interactions in Pressure-Particle Velocity Space . . . . . . . 11

2.3 Comparison of release curve from wavecode modeling with curves from various numbers of terms in expansion of Eulerian modulus about Hugoniot pressure . . . . . . . . . . . . . ... . . . . . . . . 15

2.4 Comparison of release curve from wavecode modeling with curves from various numbers of terms in the expansion of $P_{0}(V)$ about the Hugoniot volume. 16

2.5 Idealized characterization of an experimentally derived family of Hugoniot and release curves . . . . . . . . . . . . . . . . . . . 18

3.1 Axial modulus $K_{H}$ and its pressure derivatives along the release, plotted against Hugoniot pressure for MJ-2 grout . . . . . . . . . . . 21

3.2 Comparison of test-by-test MJ-2 release curves with releases calculated from algorithm for aluminum buffer experiments . . . . . . . . . . . 23

3.3 Comparison of test-by-test MJ-2 release curves with releases calculated from algorithm for tantalum buffer experiments . . . . . . . . . . . 24

3.4 Values of the axial modulus, $K_{s}$, and its pressure derivative along the release, $K_{s}^{\prime}$, plotted against Hugoniot pressure for MJ-2, several NTS tuffs, dry and water-saturated Indiana limestone $(\phi=20 \%)$, and aluminum. .

3.5 Hugoniot and release curves for two MJ-2 tests, one aluminum test and one dry limestone test, showing contrasting behavior also reflected in modulus data . . . . . . . . . . . . . . . . . 27

4.1 Schematic of the gauge problem . . . . . . . . . . . . . . . 30

4.2 Errors introduced by using single-shock MJ-2 Hugoniot for double-shock phenomena.

Errors introduced by using single-shock MJ-2 Hugoniot for shock-release phenomena: Shock propagating from MJ-2 into PMMA. . . . . . . 34

4.4 MJ-2 releases and reshocks from 10, 30, 60 and $90 \mathrm{GPa}$ : Graphic inierpretation of how the single-shock approximation gives rise to errors . . . . 


\section{Introduction}

Many applications for dynamical properties of materials require a knowledge both of the Hugoniots and the release equations-of-state of these materials. For example, groundshock attenuation rates depend on the amount of irreversible work performed on the rock mass, which at a given point in space may be expressed as the area between the Rayleigh line and the release curve in pressure/volume space.

In the past, several groups have performed tests yielding Hugoniot states and release trajectories on geological and similar materials. For example, Rosenberg et al. (1968) performed several tests on NTS tuffs and fused quartz using the immersed foil (magnetic pickup), free surface velocity (tilt-mirror), shock pressure (transducer wire) and shock velocity (streak camera and inclined mirror) methods. Explosive drivers and magnetic pickups have been used by Peterson et al. (1970) to characterize alluvium, novaculite and tonalite, and by Murri et al. (1975) to characterize carbonates and tuffs. Larson and Anderson (1979) have produced evidence for time-dependent behavior in water-saturated porous tuffs and limestones loaded by a gas gun, using magnetic particle velocity gauges to monitor loading and unloading behavior.

Over the last several years, our department has performed a variety of experiments yielding both Hugoniot states and release trajectories from each test. These tests have made use of a reverse-ballistic geometry (see Chapter 2) and velocity interferometry: and have been performed principally on geologic materials such as bedded zeolotized tuffs from the P- and N-Tunnels of the Nevada Test Site (Furnish, 1990a), Indiana Limestone (water-saturated and dry; Furnish, 1990b,c), Mini-Jade 2 (MJ-2) grout (Grady and Furnish, 1988) and granitics and tuffs related to the Joint Verification Experiment.

The results of these tests have been reduced to sets of Hugoniot states and release curves, all of which may be expressed in pressure/volume space. By assuming the validity of a Riemann integration to convert the release curves to pressure/particle velocity (Jeanloz and Ahrens, 1979, discuss the criteria for this validity and conclude that it holds for geological materials under the present conditions), the results may be written in terms of particle velocity or specific energy versus pressure. The data are still, however, discrete points and curves. There is no prescription for applying them to problems involving arbitrary or continuously varying Hugoniot states. Further computing using the experimental data may proceed by one of three routes:

1. The experimental waveforms may be used to benchmark codes. This requires computationally reproducing the experiments, including constructing equations of state for all of the ancillary materials and using the proper test geometries. 
2. The derived Hugoniot and release states may be used to benchmark codes. This approach decouples the analysis from the detills of the test geometries.

3. An empirical prescription may be prepared frorn the derived Hugoniot and release states. This is analogous to fitting Hugoniot data in $U_{s} / U_{p}$ space. Once such a prescription is prepared, experimentally-based Hugoniot and release trajectory data may be calculated and applied to problems which may require them. This approach produces results slightly removed from the direct data, but more immediately useful for problem solving.

The goal of the present report is to present an analysis in the spirit of the third approach. This analysis is applied to MJ-2 grout, a rock simulating grout. For purposes of comparison, the analysis is also applied to Indiana Limestone (dry and water-saturated), several Nevada Test Site tuffs, and aluminum. The aluminum is known to have a release above the Hugoniot (higher pressure for given density), while we have found that the other materials discussed all have releases lying below the Hugoniot. We then use the results - to analyze the problem of what errors are introduced by making the common assumption that the pressure/density release curve will lie along the Hugoniot in interpreting the output of field velocity gauges. 


\section{Developing a prescription for interpolating release data between tests}

\subsection{Summary of Experinental Technique}

This section provides a brief surnmary of the experimental techniques used to obtain the dynamic loading and unloading data used as the basis of our analysis. More detail is presented in Grady and Furnish (1988) and Furnish (1990a,b,c). An understanding of the experimental configuration is helpful, although not essential, for understanding the subsequent developments in Section 2.2.

The experimental configuration used in the present study was recently developed by Grady and Furnish (1988) for obtaining Hugoniot siates and release curves for watersaturated materials. It is shown in Figure 2.1. This type of experiment is referred to as a reverse-ballistic test because the sample material is mounted in the projectile rather than in the target assembly. When both Hugoniot and release EOS measurements are sought, this configuration is desirable. The evolution of the shock and release waves can be understood from the position-time diagram in Figure 2.1 and the pressure-particle velocity diagram in Figure 2.2. Hugoniot states are computed by impedance match techniques, where the measured quantities are projectile velocity, the velocity of the broad plateau in the wave profile (Figure 2.1, lower right subfigure) and the initial density of the sample.

Some of the tests utilized slightly different configurations. The buffers in some of the MJ-2 grout experiments were tantalum racher than aluminum (to ach eve higher Hugoniot stresses), and buffers, cups and windows in some of the limestone experiments were made of fused silica. Additionally, two of the dry limestone experiments used no cups. These changes introduced minor variations to the analysis techniques summarized here. For specifics, the reader is directed to the pertinent reports (Grady and Furnish, 1988; Furnish, 1990a,b,c)

For calculating release curves, the tests were rnodeled with the one-dimensional wavecode WONDY V (Kipp and Lawrence, 1982). Input parameters for each experiment included geometric descriptors, material descriptors for the ancillary materials and model descriptors for the sample. The geometric information required included the thickness of each plate and number of zones used for each plate, and the projectile velocity. The ancillary materials (aluminum, tantalum, lithium flouride and foam) were modeled as elastic-plastic Mie-Grüneison solids, with material descriptions including such equationof-state parameters as Hugoniot descriptions $\left(C_{o}, \mathrm{~S}\right.$ and $\left.\rho_{o}\right)$, Poisson's ratio, yield strength behavior, Grüneison gamma and fracture stresses (Table 2.1). 


\section{Equipment Configuration}

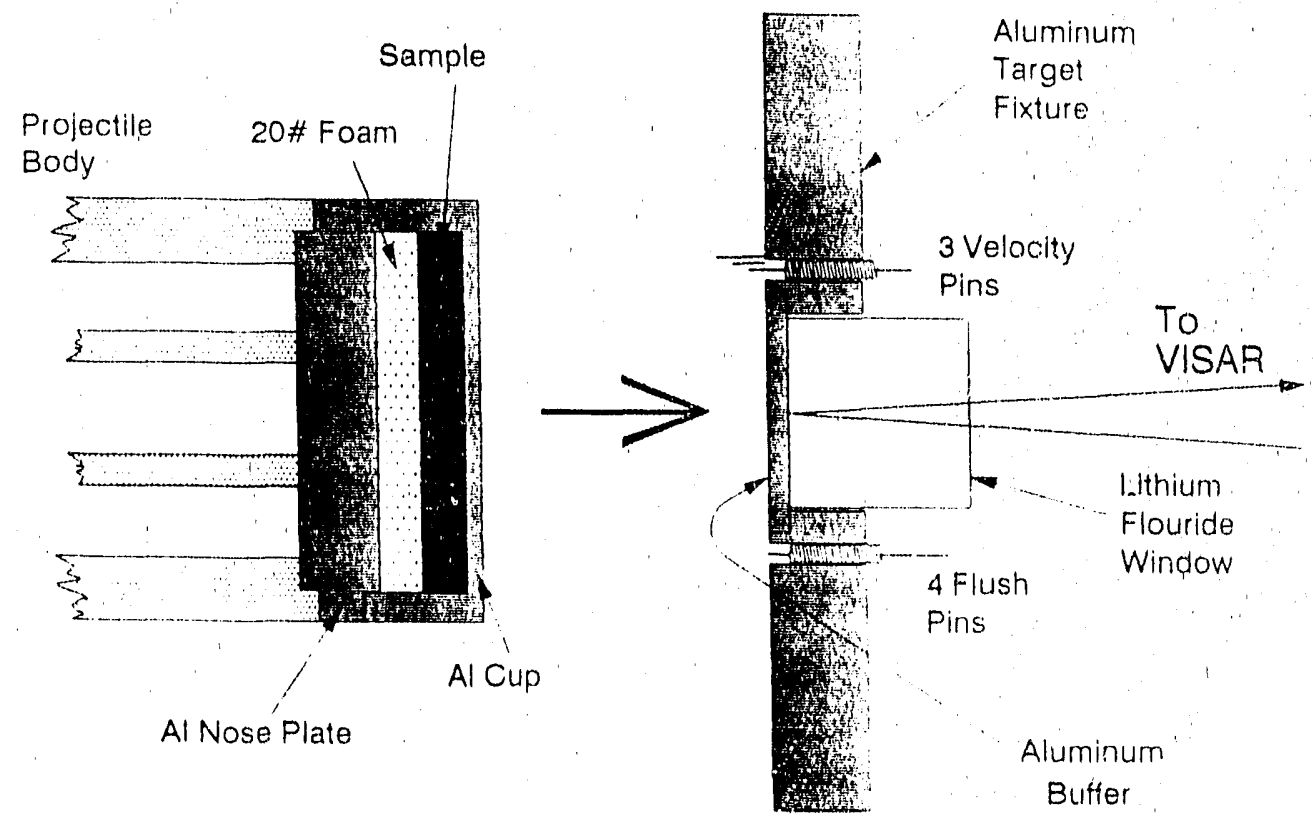

Wave Interactions

Observed Velocity Histoiy

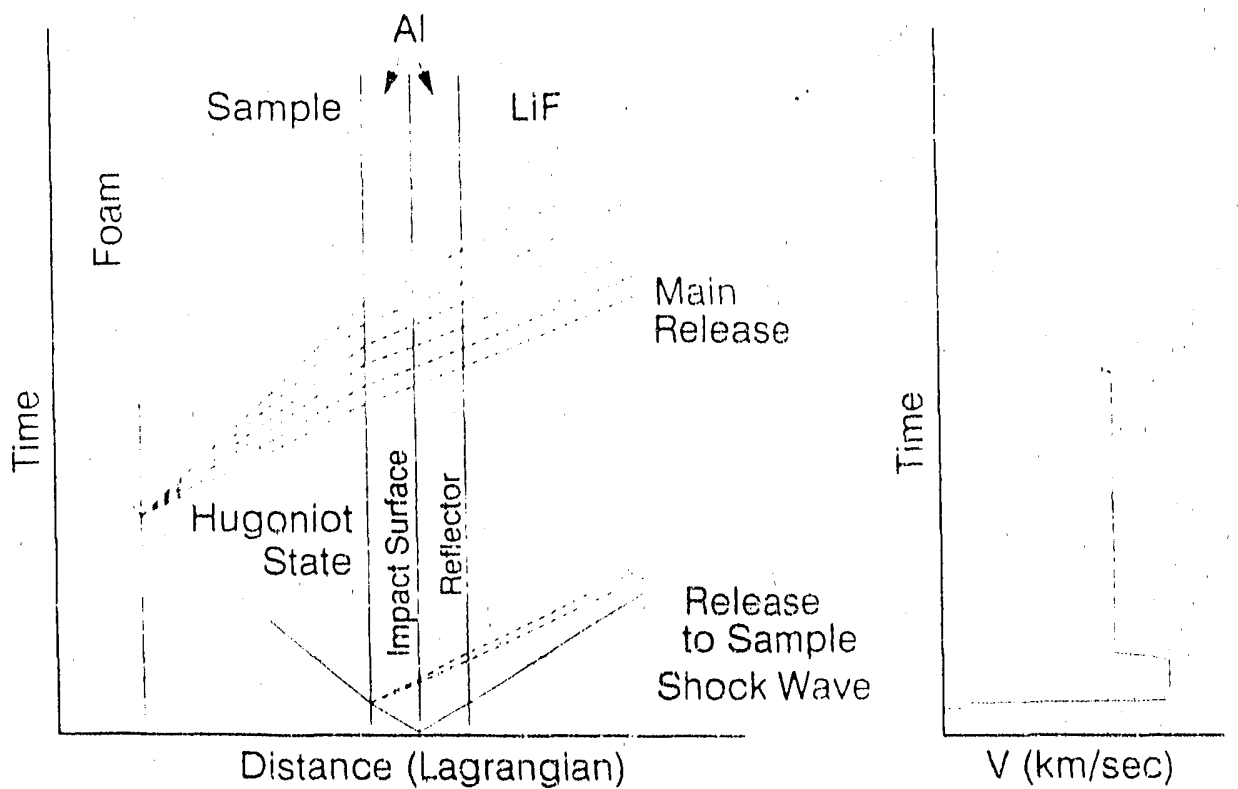

Figure 2.1. Experimental configuration used. (Top) Diagram of the configuration for a powder gun test. The long "V" entering from the right represents the laser beam entexing, being diffusely reflected, and travelling toward the VIS.AR (velocity interferometer). (Bottom Left) Wave interactions shown as Lagrangian position (coordinates based on the undeformed objects) versus time. (Bottom right) Velocity history at the monitored interface corresponding to the wave interactions discussed above. 
Calculating Hugoniot Conditions

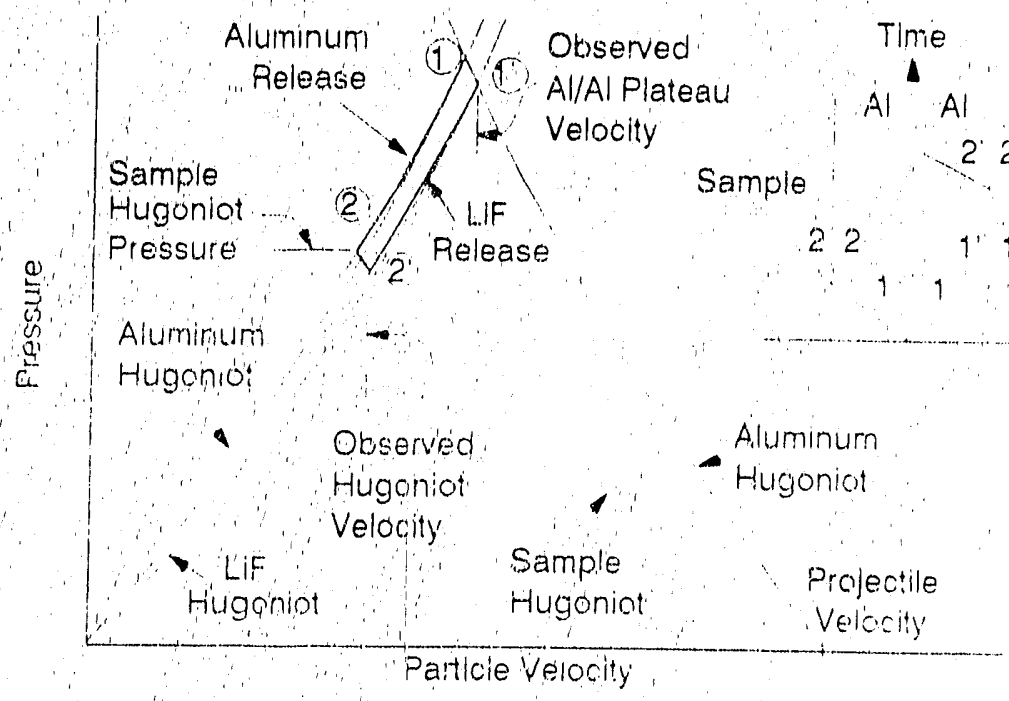

Figure 2:2. Wave interactions in pressure-particle velocity space. Inset gives position versus time location of the numbered states in the main figure.

The samples were modeled as hydrodynamic Mie-Grüneison solids for the loading wave (characterized by the Hugoniot parameters $C_{c}, S$ and $\rho_{o}$ ). For relatively weak materials such as tuffs, limestones and grouts (strengths less than about $1 \mathrm{kbar}$ ), this is a reasoriable assumption. For a competent granite or quartzite, this assumption might need to be modified.

The unloading behavior is represented as an axial Lagrangian modulus depending on pressure (or, to be completely correct, axial stress) according to:

$$
B_{s}=V_{c} \frac{d P}{d V} l_{s}=B_{0}\left[1+B_{1}\left(\frac{P}{P_{H}}-1\right)+B_{2}\left(\frac{P}{P_{H}}-1\right)^{2}+B_{3}\left(\frac{P}{P_{H}}-1\right)^{3}\right] .
$$

Here, $P_{H}$ is the Hugoniot pressure and the $B_{i}$ parameters $(i=0,1,2,3)$ are adjustable. This modulus is used to compute incremental pressures.

Operationally, the modull are derived by a process of making progressively bettes guesses of the parameters $B_{i}$. The proper Hugoniot conditions are derived by the impedance match analysis mentioned above, so are known at the beginning of this process. A typical rurin includes roughly 800 zones and requires 8-15 minutes of CPU time 
on a VAX 8700 computer. The release curves can be extracted to the precision of this technique in 5-10 runs of the wavecode. The quantity $B_{0}$, which corresponds to the Lagrangian sound velocity at the Hugoniot state, can be calculated from the explicit sound velocity calculations described in Grady and Furnish (1988) and Furnish (1990a). An error in $B_{0}$ will give an error in the timing of the first arrival of the release wave. Generally it is necessary to adjust $B_{0}$ slightly to give an optimal fit; this is mainly due to perceptual considerations in "eyeballing" a best fit. The coefficients $B_{1}, B_{2}$ and $B_{3}$ are then adjusted to give the proper slope and shape to the calculated release wave. The results of each calculation are compared with the experimental velocity history and the iteration continued until adequate agreement is achieved.

\subsection{Expressing the release curves as Eulerian moduli and pres- sure derivatives at the Hugoniot condition}

The method we shall pursue for generalizing a set of release paths is to describe how the axial release modulus and its pressure derivatives (at the Hugoniot pressure) behave as a function of Hugoniot pressure. Knowing these quantities for an arbitrary Hugoniot pressure gives us the Taylor expansion about the Hugoniot pressure of the axial inodulus; integrating gives the pressure-volume curve of the release. First we need to look at how a single test is reduced to this modulus and its pressure derivatives; later we will couple together all of the tests.

Table 2.1. Equation of State Properties ${ }^{\dagger}$

$\begin{array}{lrcccc}\text { Material } & \begin{array}{c}\rho_{0} \\ \left(\mathrm{~kg} / \mathrm{m}^{3}\right)\end{array} & \begin{array}{c}\mathrm{C}_{0} \\ (\mathrm{~m} / \mathrm{s})\end{array} & \mathrm{S} & \gamma_{0} & \begin{array}{c}\mathrm{Y} \\ (\mathrm{GPa})\end{array} \\ \begin{array}{l}\text { Lithium } \\ \text { fluoride }\end{array} & 2,641 & 5,148 & 1.35 & 1.63 & 0.2 \\ \text { Aluminum } & 2,698 & 5,370 & 1.34 & 2.1 & 0.3 \\ \text { Tantalum } & 16,680 & 3,293 & 1.31 & 1.60 & 0.7 \\ \text { Polyurethane } & 1,265 & 2,486 & 1.58 & 1.55 & -\end{array}$

1 EOS properties are appropriate for a Grüneisen model with a linear shock velocityparticle velocity behavior.

- Properties are for solid polyurethane. A model must account for collapse from the distended density corresponding to 20 or 40 pound foam (e.g., Hermann, 1968). 
As discussed above, the particular version of the WONDY V wavecode used here models the sample as a Mie-Grüneison material under loading to the Hugoniot state, then (for release or further loading) as having a Lagrangian axial modulus $B_{s}$, which may be expressed as an expansion about $P_{H}$ (the Hugoniot pressure). It may be related to the Eulerian modulus $K$, as:

$$
K_{0} \equiv-\left.V \frac{\partial P}{\partial V}\right|_{0}=\frac{V}{V_{o}} \cdot B_{0}=\frac{\rho_{o}}{\rho} \cdot B_{0} .
$$

At the Hugoniot pressure, this is:

$$
K_{\bullet}\left(P_{H}\right)=\frac{\rho_{o}}{\rho_{H}} \cdot B_{0} .
$$

The first pressure derivative $K_{a}^{\prime}$ (note - along the release) is:

$$
\begin{gathered}
\left.K_{\mathrm{s}}^{\prime} \equiv \frac{\partial K_{\mathrm{o}}}{\partial P}\right|_{\mathrm{s}}=\frac{\rho_{\mathrm{o}}}{\rho} \cdot\left[B_{\mathrm{o}}^{\prime}-\frac{B_{\mathrm{o}}}{K_{\mathrm{o}}}\right] \\
\Rightarrow K_{\mathrm{s}}^{\prime}\left(P_{H}\right)=\frac{\rho_{0}}{\rho_{H}} \cdot \frac{B_{0} B_{1}}{P_{H}}-1 .
\end{gathered}
$$

The second pressure derivative $K_{3}^{\prime \prime}$ is:

$$
\begin{gathered}
\left.K_{s}^{\prime \prime} \equiv \frac{\partial K_{a}^{\prime}}{\partial P}\right|_{s}=\frac{\rho_{o}}{\rho} \cdot \frac{1}{K_{s}}\left[K_{s} B_{s}^{\prime \prime}-2 B_{s}^{\prime}+\frac{B_{s}}{K_{s}}\left(K_{s}^{\prime}-1\right)\right] \\
\Rightarrow K_{s}^{\prime \prime}\left(P_{H}\right)=-\frac{B_{1}}{P_{H}}+2 \frac{\rho_{o}}{\rho_{H}} \cdot \frac{B_{0} B_{2}}{P_{H}^{2}} .
\end{gathered}
$$

The third pressure derivative $K_{a}^{\prime \prime \prime}$ is:

$$
\begin{aligned}
& \left.K_{a}^{\prime \prime \prime} \equiv \frac{\partial K_{a}^{\prime \prime}}{\partial P}\right|_{a}=\frac{\rho_{0}}{\rho} \cdot \frac{1}{K_{a}^{2}}\left[B_{s} \cdot \frac{1}{K_{0}}\left(K_{s} K_{a}^{\prime \prime}-2 K_{s}^{\prime 2}-3 K_{a}^{\prime}-1\right)+B_{a}^{\prime}\left(3 K_{a}^{\prime}+3\right)+B_{a}^{\prime \prime}\left(-3 K_{0}\right)+B_{a}^{\prime \prime \prime}\right] \\
& \Rightarrow K_{s}^{\prime \prime \prime}\left(P_{H}\right)=\frac{1}{P_{H}^{3}} \cdot\left(B_{1}^{2} P_{H}-4 B_{2} P_{H}+6 \frac{\rho_{o}}{\rho_{H}} \cdot B_{0} B_{3}\right) .
\end{aligned}
$$

Note that $K_{a}^{\prime \prime \prime}$ can be nonzero even if $\mathrm{B}_{3}=0$. Also, all of these derivatives evaluated at the Hugoniot may be expressed in terms of $P_{H}$, the four $B_{i}$ and the ratio $\rho_{0} / \rho_{H}$. 
How well does the Taylor expansion of the Eulerian axial modulus about $P_{H}$ to this order describe the release curve behavior? Is it an adequate power-series representation of the release curve? The release curve corresponding to this modulus is computed as:

$$
\begin{gathered}
V(P)=V(P=0) \cdot \int_{P}^{P_{H}} \frac{d P}{B_{\bullet}(P)} \\
=V\left(P_{H}\right) \cdot \exp \left[\int_{P_{-} P_{H}}^{0} \frac{d \xi}{K_{\iota}(\xi)}\right] \\
=V\left(P_{H}\right) \cdot \exp \left[\int_{P_{-} P_{H}}^{0} \frac{d \xi}{K\left(P_{H}\right)+\xi K_{0}^{\prime}\left(P_{H}\right)+\frac{1}{2} \xi^{2} K_{s}^{\prime \prime}+\frac{1}{6} \xi^{s} K_{s}^{\prime \prime \prime}}\right] .
\end{gathered}
$$

Fig. 2.3 provides a comparison of the curve directly from the wavecode model with curves calculated using various numbers of terms in the expansion. The example here used a sample of a tuff from the Nevad: Test Site. The solid curve ("calculated for $B_{0}$ through $\mathrm{B}_{3}$ ") lies almost on the output from the computer modeling (traced by symbols $x$ ); the small descrepancy is due chiefly to imperfections in the wavecode calculation of the Hugoniot condition. The other three curves should be compared with the solid curve for goodness-of-fit determinations. We see that using the first four terms of the Taylor series (for $K_{s}$ and the first three pressure derivatives) reproduces the release curve very well; using less terms does not.

\subsection{Attempting to express the release curves in terms of vol- ume derivatives of pressure}

Several of the major material dynamics computer codes currently in use have volume and energy as the principal independent variables. In an attempt to provide EOS coefficients directly useable in those codes, I computed the quantities $\left.\left(\partial^{n} P / \partial V^{n}\right)\right|_{s, H}$ ( $n \in\{0,1,2,3,4\})$ in terms of the $B_{i}$ already described. Here, the subscripts mean that the derivative is evaluated for an isentrope at the Hugoniot condition. Four derivatives are needed here instead of the three used earlier because the moduli used in the earlier technique are themselves derivatives of $V_{s}(P)$. These derivatives are

$$
\begin{gathered}
\left.P\right|_{H}=P_{H}, \\
\left.\frac{\partial P}{\partial V}\right|_{, H}=-\frac{B_{0}}{V_{0}}, \\
\left.\frac{\partial^{2} P}{\partial V^{2}}\right|_{s, H}=\frac{B_{0}^{2}}{V_{0}^{2} P_{H}} B_{1},
\end{gathered}
$$




$$
\begin{aligned}
& \left.\frac{\partial^{3} P}{\partial V^{3}}\right|_{g, H}=-\frac{B_{0}^{3}}{V_{0}^{3} P_{H}^{2}}\left(B_{1}^{2}+2 B_{2}\right), \text { and } \\
& \left.\frac{\partial^{4} P}{\partial V^{4}}\right|_{e, H}=\frac{B_{0}^{4}}{V_{0}^{4} P_{H}^{3}}\left(B_{1}^{3}+8 B_{1} B_{2}+6 B_{3}\right) .
\end{aligned}
$$

The release curve may be expressed in terms of these derivatives as a Taylor series according to

$$
P_{\mathrm{rel}}=P_{0}(V)=P_{H}+\left.\frac{\partial P}{\partial V}\right|_{s, H}\left(V-V_{H}\right)+\ldots+\left.\frac{1}{n !}\left(\partial^{n} P / \partial V^{n}\right)\right|_{\mathrm{Q}, H}\left(V-V_{H}\right)^{n}+\ldots
$$

Unfortunately, this series does not converge rapidly to the original function $P_{s}(V)$, as shown in Figure 2.4. It is not an adequate power series representation of the release

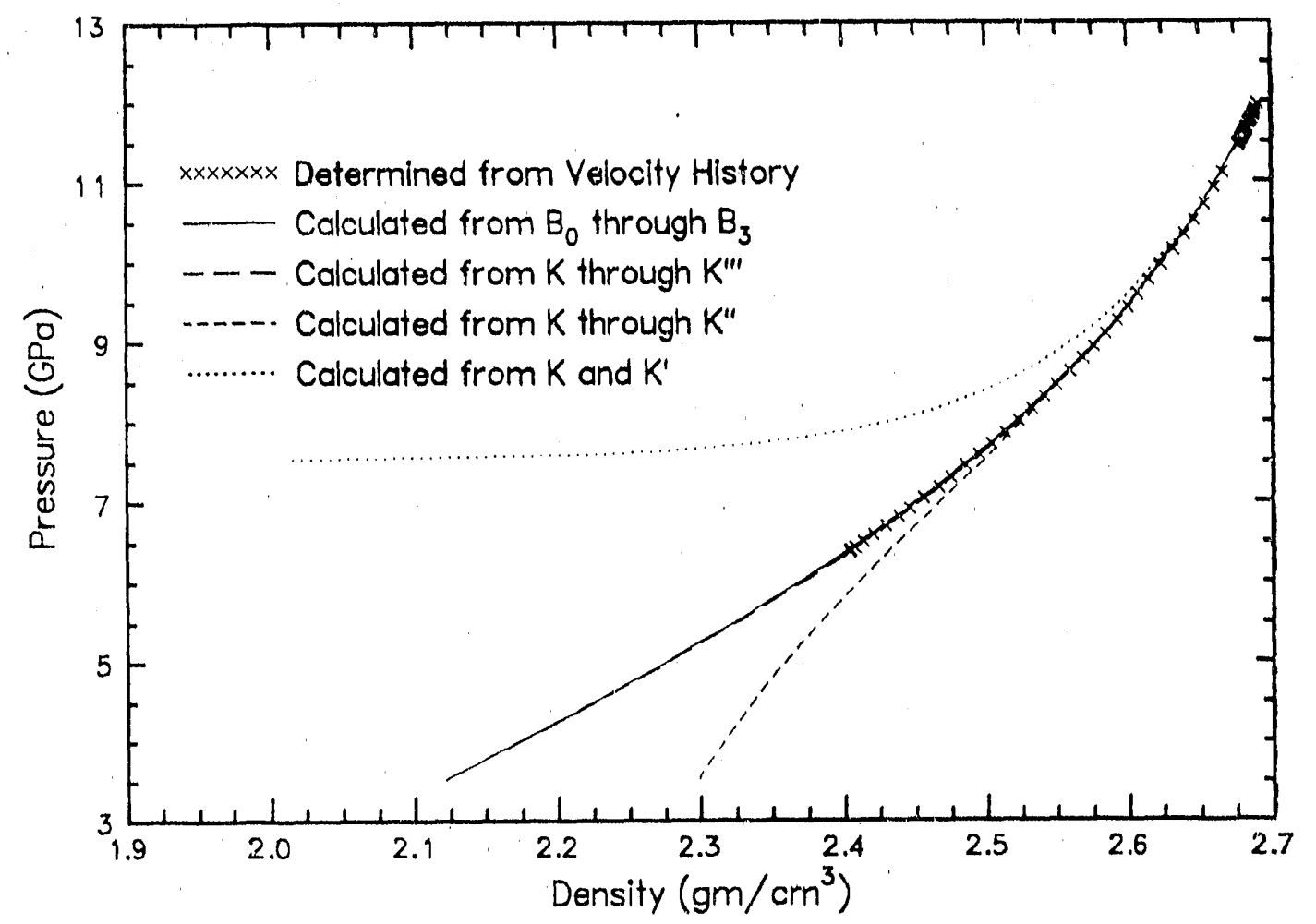

Figure 2.3. Comparisen of release curve from wavecode modeling with curves from various numbers of terms in expansion of Eulerian modulus about Hugoniot pressure. The curve determined from the velocity history extends downward to $6.4 \mathrm{GPa}$, but the other curves are continued farther for illustration purposes. This particular example is from a test with the MC-2 unit of the Paintbrush Tuff (Nevada Test Site). 
trajectories for any reasonable number of derivatives used. Other calculations of power series representations of these curves using $\mathrm{V}$ as an independent variable (eg. least-squares fits to the entire curve) have also failed to produce adequate power-series representations of the release curves. We therefore abandon this approach for the present.

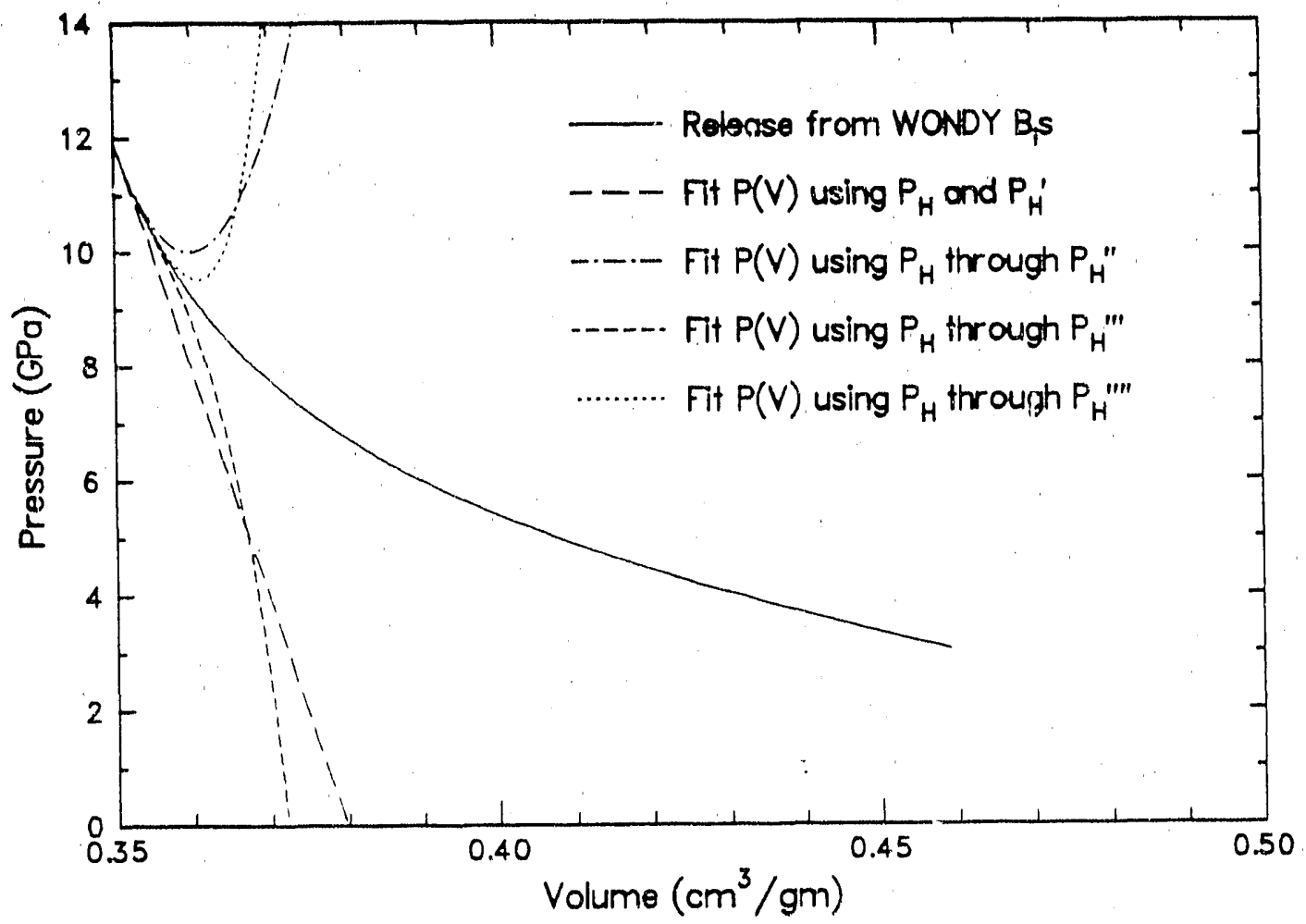

Figure 2.4. Comparison of release curve from wavecode modeling with curves from various numbers of terms in the expansion of $P_{s}(V)$ about the Hugoniot volume.

\subsection{Linking the modulus data together into the desired algo- rithm}

If the material under study behaves perfectly, and the experiments are conducted in such a manner as to have no sources of error, both the Hugoniot and the release properties of the shots will be completely consistent from one test to the next. We may plot up the results of such a set of experiments, say, the Hugoniot as particle velocity $U_{p}$ versus shock velocity $U$, and versus pressure, and the releases as the axial Eulerian modulus $K \equiv \partial P / \partial V$ and its first three pressure derivatives versus Hugoniot pressure. The results will look somewhat like the plots in Figure 2.5.

Clean curves may be drawn through all of the points. Phase transitions may appear, and will give rise to well-formed discontinuities in the fit curves or in their derivatives. From these curves, we will be able to predict quite well the results of some other test 
at some arbitrary IIugoniot pressure in the range of the tests performed. It will be possible to write down the Hugoniot conditions and the axial modulus and its first three pressure derivatives at those Hugoniot conditions. From this information, we will be able to proceed according to the methods of the previous section and produce a release curve centered at that new Hugoniot condition. This is the algorithm sought.

The curves drawn through the points on each of the plots in Figure 2.5 are only intended to give interpolation schemes for the coordinates of those plots. The reader should remember, for example, that the derivative of $K$ with respect to Hugoniot pressure does not necessarily have any relation to $K^{\prime}$, which is the derivative of $K$ with respect to pressure along the release. 


\section{Hugoniot}
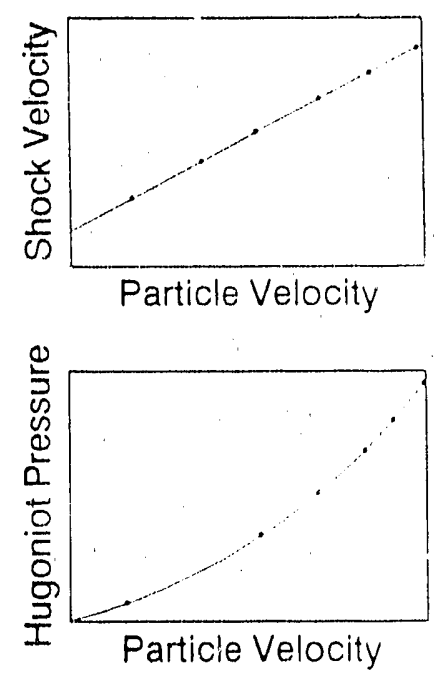

Release EOS
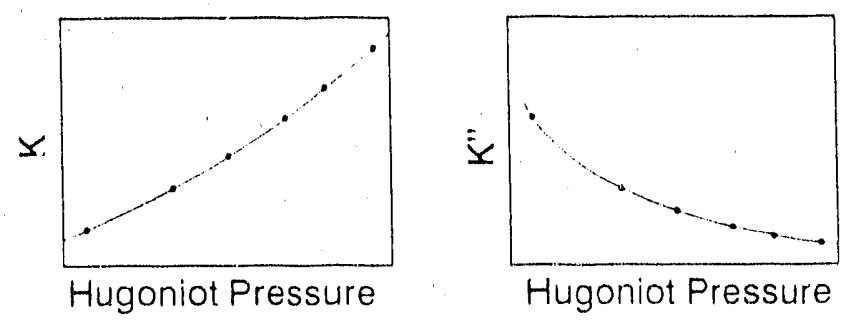

Hugoniot Pressure
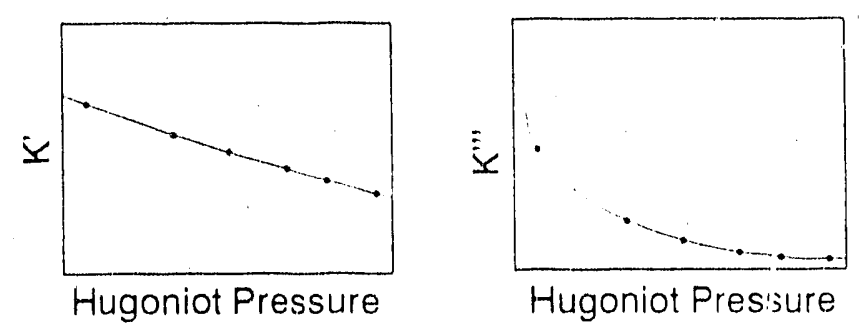

Figure 2.5. Idealized characterization of an experimentally derived family of Hugoniot and release curves 


\section{Empirical EOS summaries for specific materials}

This chapter presents the results of applying the analyses of Chapter 2 to several materials which have been recently tested in our department. The MJ-2 grout results are discussed in detail, and results from Indiana Limestone (18\% porosity; water saturated and dry) and NTS tuffs are presented briefly as illustrations of variability. Finally, the results of several earlier tests on aluminum have been re-examined and cast into the form required for the present analysis. Aluminum is the only material amongst those discussed here which does not have a release lying below the Hugoniot.

\subsection{Mini Jade 2 Grout}

The equation of state of Mini Jade 2 (MJ-2) grout (Hugoniot and release) was evaluated to $70 \mathrm{GPa}$ by Grady and Furnish (1988). This rock-simulating grout was designed for use as a rock-simulating grout for groundshock measurements in nuclear testis.

Table 3.1 summarizes the $B_{i}\left(B_{0}, B_{1}, B_{2}\right.$ and $\left.B_{3}\right)$ and the axial modulus $K_{\text {s }}$ and its pressure derivatives for all of the reverse-ballistics tests we have conducted on $\mathrm{MJ}$ 2 grout. Figure 3.1 shows plots of the axial modulus and pressure derivative versus Hugoniot pressure for these tests.

One complication is an artifact of the way we chose to extract the initial release curves for the MJ-2. We chose to model the grout according to a Hugoniot piece-bypiece linear in $U_{\bullet} / U_{p}$ space for the computational simulations instead of using shot-byshot Hugoniot points. The question is whether it is valid to use the actual shot-by-shot values for the Hugoniot pressure in the expansion of $B$, about $\mathrm{P}_{\mathrm{H}}$. Hence we computed the $B_{i}$ (and hence the moduli) two ways. The first used the actual shot-by-shot values for the Hugoniot pressure in the expansion of $B_{s}$ about $P_{H}$. The Hugoniot was, however, represented in the conventional fit form expressed piece-by-piece in terms of $\mathrm{C}_{0}, \mathrm{~s}$ and $\rho_{0}$. The second, which is more internally consistent when using a fit Hugoniot, involved calculating the Hugoniot pressures each test would have given for the measured projectile velocity with the fit Hugoniot describing the sample, then modifying the individual coefficients $B_{i}$ to be consistent with that pressure Both results are plotted in Figure 3.1. The results from the second are tabulated in Talle 3.1; for the $B_{i}$ coefficients used in the first method, see Grady and Furnish (1988).

In subsequent tests (including all of the others discussed in this report) we have not used fir Hugoniots until later in the analysis, but we do not believe that the benefit of redoing the MJ-2 analyses by the newer method would be worth the large effort required. 
Table 3.1. Coefficients $B_{i}$ for MJ-2 using a smoothed Hugoniot. All coefficients have been corrected to Hugoniot conditions obtained with model Hugoniots: $\mathrm{C}_{0}=2.4, \mathrm{~S}=1.6\left(\mathrm{P}_{\mathrm{H}} \leq 20 \mathrm{GPa}\right) ; \mathrm{C}_{0}=2.4, \mathrm{~S}=1.36\left(\mathrm{P}_{\mathrm{H}}>20 \mathrm{GPa}\right)$. The jump represents an apparent phase transition in the material.

\begin{tabular}{|c|cccc|cccc|}
\hline $\begin{array}{c}\text { Test } \\
\#\end{array}$ & $\begin{array}{c}\mathrm{B}_{0} \\
(\mathbf{G P a})\end{array}$ & $\mathrm{B}_{1}$ & $\mathrm{~B}_{2}$ & $\mathrm{~B}_{3}$ & $\begin{array}{c}\mathrm{P}_{\mathrm{H}} \\
(\mathrm{GPa})\end{array}$ & $\begin{array}{c}\mathrm{U}_{\mathrm{P}} \\
(\mathrm{km} / \mathrm{sec})\end{array}$ & $\begin{array}{c}\mathrm{U}_{\mathbf{8}} \\
(\mathrm{km} / \mathbf{s e c})\end{array}$ & $\begin{array}{c}\rho_{\mathrm{H}} \\
\left(\mathrm{Mg} / \mathrm{m}^{3}\right)\end{array}$ \\
\hline 1 & 53.5638 & 1.8627 & 1.9659 & 0.7223 & 4.201 & 0.619 & 3.391 & 2.4470 \\
2 & 69.6831 & 1.3979 & 0.5988 & 0.0000 & 6.180 & 0.829 & 3.727 & 2.5723 \\
3 & 96.9855 & 1.2500 & 0.2500 & -0.1000 & 8.349 & 1.039 & 4.049 & 2.6830 \\
4 & 114.7360 & 1.4975 & 1.1956 & 0.7483 & 10.234 & 1.189 & 4.303 & 2.7639 \\
5 & 114.8382 & 1.0878 & 0.2933 & 0.0000 & 11.959 & 1.324 & 4.518 & 2.8288 \\
\hline 6 & 112.4721 & 0.9004 & -0.0014 & -0.1010 & 12.056 & 1.331 & 4.529 & 2.8322 \\
7 & 139.6131 & 1.2987 & 0.4993 & 0.0000 & 15.098 & 1.548 & 4.877 & 2.9300 \\
8 & 187.6341 & 1.8262 & 1.5302 & 0.5101 & 18.519 & 1.770 & 5.232 & 3.0225 \\
\hline 13 & 273.7085 & 1.7929 & 0.9937 & 0.0985 & 24.696 & 2.257 & 5.470 & 3.4053 \\
14 & 313.4344 & 1.9497 & 2.0147 & 0.9095 & 28.686 & 2.483 & 5.777 & 3.5076 \\
15 & 355.7445 & 1.8395 & 1.5907 & 0.3970 & 35.312 & 2.827 & 6.245 & 3.6544 \\
16 & 524.8220 & 2.0055 & 1.6055 & 0.4018 & 43.108 & 3.195 & 6.746 & 3.8000 \\
\hline 18 & 522.0742 & 1.9898 & 1.2898 & 0.1966 & 46.565 & 3.348 & 6.954 & 3.8574 \\
19 & 777.9760 & 2.0062 & 1.6062 & 0.4021 & 56.671 & 3.767 & 7.523 & 4.0057 \\
20 & 787.3456 & 1.9904 & 1.5904 & 0.3968 & 65.068 & 4.088 & 7.959 & 4.1116 \\
\hline
\end{tabular}

\begin{tabular}{|c|cccc|}
\hline $\begin{array}{c}\text { Test } \\
\#\end{array}$ & $\begin{array}{c}\mathrm{K}_{\mathrm{H}} \\
(\mathrm{GPa})\end{array}$ & $\mathrm{K}_{\mathrm{H}}^{\prime}$ & $\begin{array}{c}\mathrm{K}_{\mathrm{H}}^{\prime \prime} \\
\left(\mathrm{GPa}^{-1}\right)\end{array}$ & $\begin{array}{c}\mathrm{K}_{\mathrm{H}}^{\prime \prime \prime} \\
\left(\mathrm{GPa}^{-2}\right)\end{array}$ \\
\hline 1 & 43.7796 & 18.4121 & 9.30975 & 2.3099349 \\
2 & 54.1791 & 11.2555 & 1.47282 & -0.0115508 \\
3 & 72.2954 & 9.8238 & 0.36889 & -0.0664510 \\
4 & 83.0243 & 11.1480 & 1.74915 & 0.3234802 \\
5 & 81.1930 & 6.3853 & 0.24207 & 0.0000702 \\
\hline 6 & 79.4243 & 4.9318 & -0.07623 & -0.0218455 \\
7 & 95.2977 & 7.1974 & 0.33143 & -0.0013617 \\
8 & 124.1595 & 11.2435 & 1.00940 & 0.0517077 \\
\hline 13 & 160.7545 & 10.6706 & 0.45124 & 0.0050607 \\
14 & 178.7182 & 11.1472 & 0.80720 & 0.0361429 \\
15 & 194.6935 & 9.1421 & 0.44465 & 0.0081444 \\
16 & 276.2212 & 11.8506 & 0.43077 & 0.0070220 \\
\hline 18 & 270.6889 & 10.5667 & 0.27931 & 0.0026094 \\
19 & 388.4364 & 12.7507 & 0.35313 & 0.0044012 \\
20 & 382.9854 & 10.7152 & 0.25714 & 0.0027430 \\
\hline
\end{tabular}



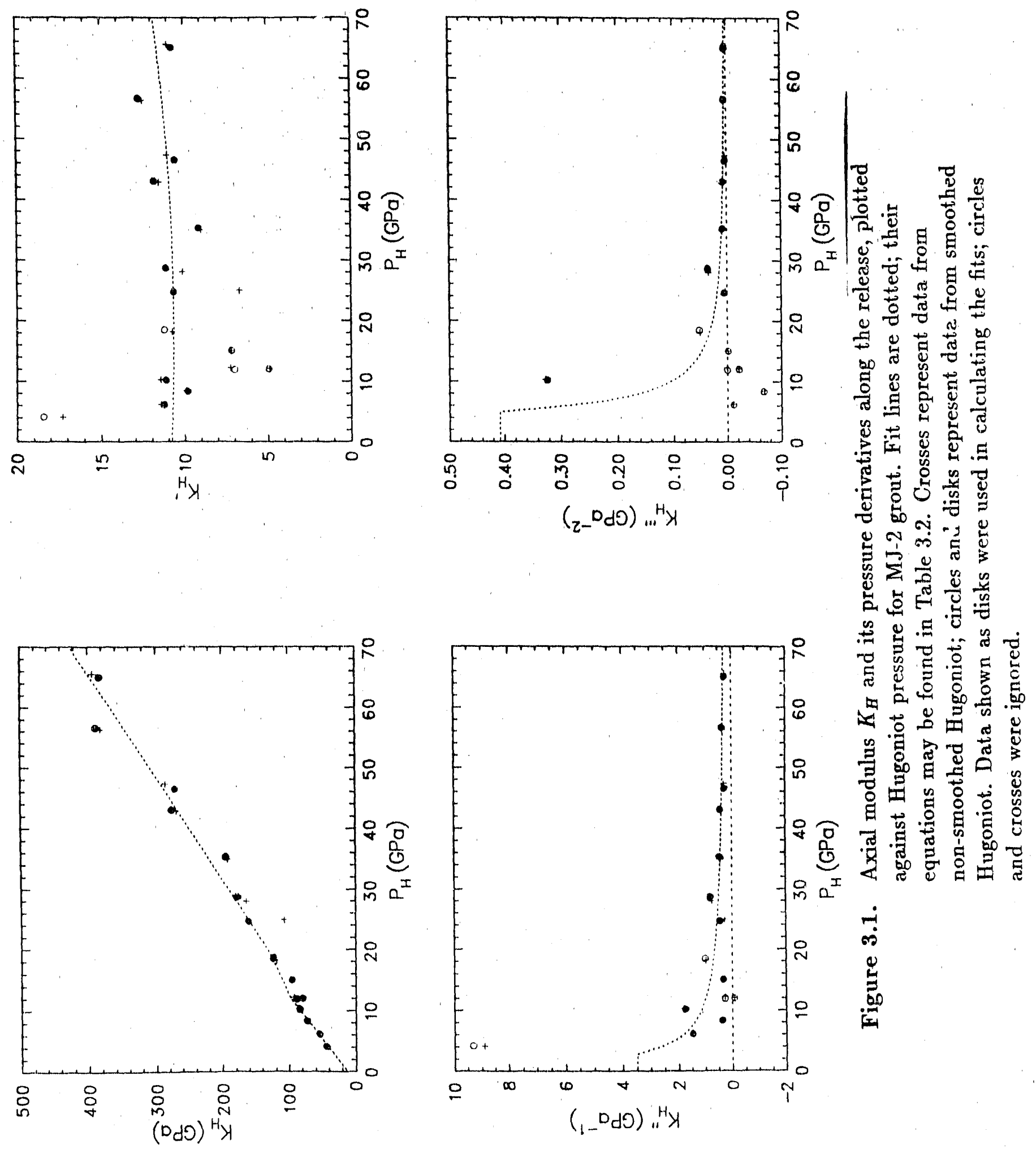
Interpolating between tests is simply a matter of drawing satisfactory fits through the data of Figure 3.1. The fits used are shown there, and enumerated in Table 3.2.

Table 3.2. Equations for fits of axial modulus and pressure derivatives versus Hugoniot pressure for MJ-2

\begin{tabular}{|c|c|c|}
\hline Modulus & Fit curve & Pressure range (GPa) \\
\hline \hline \multirow{3}{*}{$\mathrm{K}_{\mathrm{H}}$} & $\mathrm{K}_{\mathrm{H}}=11.884 \mathrm{GPa}+7.0687 \mathrm{P}_{\mathrm{H}}$ & $0 \leq \mathrm{P}_{\mathrm{H}}<12$ \\
& $\mathrm{~K}_{\mathrm{H}}=43.7884 \mathrm{GPa}+4.410 \mathrm{P}_{\mathrm{H}}$ & $12 \leq \mathrm{P}_{\mathrm{H}}<20$ \\
& $\mathrm{~K}_{\mathrm{H}}=14.6071 \mathrm{GPa}+5.874 \mathrm{P}_{\mathrm{H}}$ & $20 \leq \mathrm{P}_{\mathrm{H}}$ \\
\hline $\mathrm{K}_{\mathrm{H}}^{\prime}$ & $\mathrm{K}_{\mathrm{H}}^{\prime}=10.8064 \mathrm{GPa}-0.016707 \mathrm{P}_{\mathrm{H}}$ & $0 \leq \mathrm{P}_{\mathrm{H}} \leq 70$ \\
& $+0.0004763 \mathrm{P}_{H}^{2}$ & \\
\hline $\mathrm{K}_{\mathrm{H}}^{\prime \prime}$ & $\mathrm{K}_{\mathrm{H}}^{\prime \prime}=6.082$ & $0 \leq \mathrm{P}_{\mathrm{H}}<3$ \\
& $\mathrm{~K}_{\mathrm{H}}^{\prime \prime}=\left(10.0076+0.1488 \mathrm{P}_{\mathrm{H}}\right) / \mathrm{P}_{\mathrm{H}}$ & $3 \leq \mathrm{P}_{\mathrm{H}} \leq 70$ \\
\hline $\mathrm{K}_{\mathrm{H}}^{\prime \prime \prime}$ & $\mathrm{K}_{\mathrm{H}}^{\prime \prime}=0.4075$ & $0 \leq \mathrm{P}_{\mathrm{H}}<5$ \\
& $\mathrm{~K}_{\mathrm{H}}^{\prime \prime \prime}=\left(9.9235+0.052361 \mathrm{P}_{\mathrm{H}}\right) / \mathrm{P}_{\mathrm{H}}^{2}$ & $5 \leq \mathrm{P}_{\mathrm{H}} \leq 70$ \\
\hline
\end{tabular}

A test of the consistency of the data and the chosen fits is to use the algorithm to reproduce the original data. This is analogous to inspecting the scattering on $a U_{s} / U_{p}$ plot of Hugoniot data. Poor reproduction of a certain release suggests that either the particular experiment is anomalous or the fitting of the modulus data did not reflect the nature of that data as well as it might. This is analogous to the way that $U_{s} / U_{p}$ fits sometimes must be constructed in segments.

The modeled release curves are compared with the releases directly from the wavecode in Figures 3.2 and 3.3. The fit to $K$, has been carefully selected in the lower pressure region $(\mathrm{P} \leq 20 \mathrm{GPa}$ ) to give a reasonable match for the release curves from these pressures, with the kink occurring in the phase transition region. The poorest fits occur for experiments DNA7, DNA8 and DNA19. The poorness of these fits is probably due to a combination of difficulties with different experimental configurations (tantalum buffers were used in DNA6-8 and DNA18-20; these spall and hence give slightly less reliable release profiles) and (for DNA7 and I NA8) problems with constructing an appropriate fit for $K_{0}\left(P_{H}\right)$ in this region. Physically, the onset of the $\mathrm{Si}^{\mathrm{IV}} \rightarrow \mathrm{Si}^{\mathrm{VI}}$ (quartz-stishovite) phase transition may also affect the results for tests DNA7 and DNA8.

The goodness of fit of the releases is a much stronger function of how well $K$, was chosen than of how well $K_{\text {? }}^{\prime}$ was chosen (with successive derivatives progressively less important). We have not made a careful study to determine how much the precise choice of $K_{a}^{\prime \prime}$ and $K_{a}^{\prime \prime \prime}$ influence the goodness of fit. In trying combinations of wh $B_{i}$ coefficients in the wavecode to reproduce the experimental velocity profiles, we have found that there is a certain latitude to make compensating adjustments in $B_{3}$ and $B_{4}$, still achieving a good eyeball fit to the velocity profilk. Loosely speaking, $K_{\text {: }}^{\prime \prime}$ depends more strongly on $\mathrm{B}_{3}$ than on $\mathrm{B}_{4}$, and the reverse is true for $K_{a}^{\prime \prime \prime}$. Apparently $K_{s}^{\prime \prime}$ and $K_{s}^{\prime \prime \prime}$ are only weakly constrained in this procedure. 

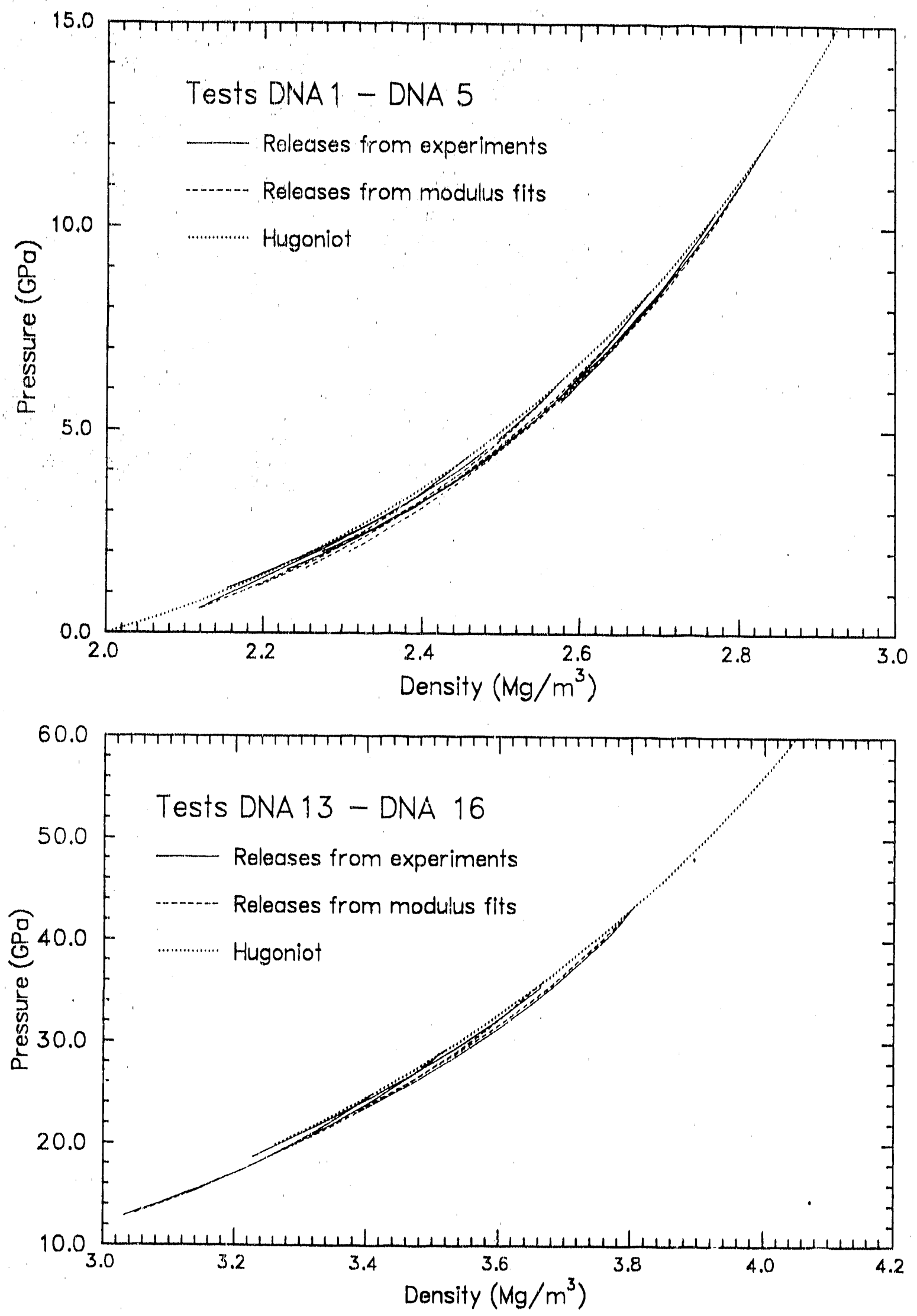

Figure 3.2. Comparison of test-by-test $\mathrm{MJ}-2$ release curves with releases calculated from algorithm for aluminum buffer experiments 

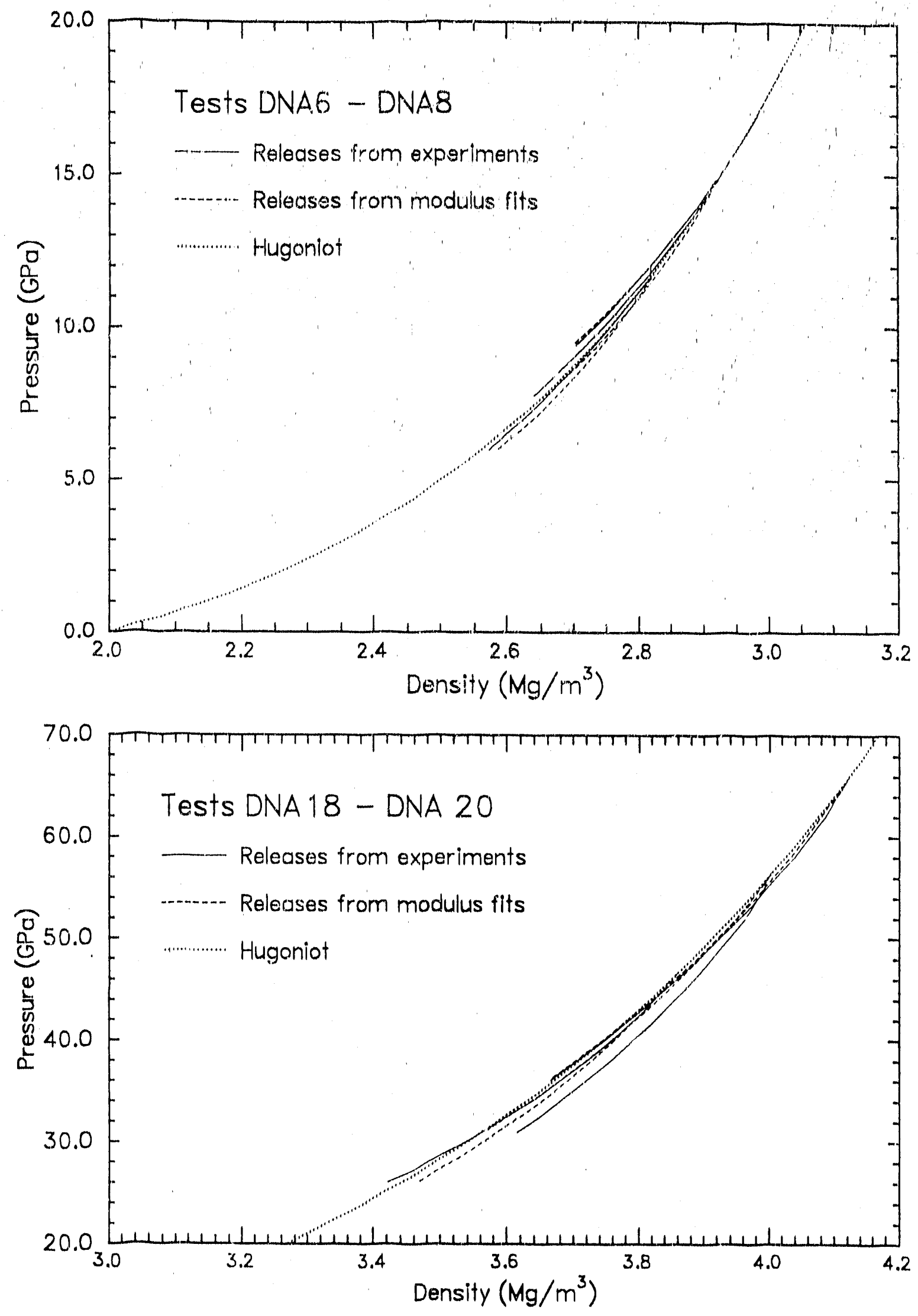

Figure 3.3. Comparison of test-by-test MJ-2 release curves with releases calculated from algorithm for tantalum buffer experiments 
In Figure 3.1, please note that if a test was not used to determine the fit curve for $K_{o}^{\prime}$, it was also skipped for $K_{s}^{\prime \prime}$ and $K_{s}^{\prime \prime \prime}$. This is for the reason just mentioned, that successive derivatives are inore poorly constrained.

This completes the analysis of our data to give a general prescription for MJ-2 release curves from Higoniot piessures anywhere in the range 4-70 GPa.

\subsection{Other materials}

We have performed dynamic compression and release tests using the techniques described in Section 2.2 for several geological materials. These include dry and watersaturated Indiana Limestone (Furnish, 1990b) and tuffs from $P$ and $N$ Tunnels at the Nevada Test Site (Furnish, 1990a). We have also obtained unpublished data from two aluminum tests performed by J. R. Asay (personal communication) which have the same geometry as the reverse-ballistics tests we have performed, and can be computationally treated the same way. It is worthwhile to briefly present the modulus data for those materials and compare them with the MJ-2 data.

Figure 3.4 shows the behavior of $K_{\text {a }}$ and $K_{s}^{\prime}$ for all of these materials. The same fits drawn in Figure 3.1 are reproduced here. The terms $K_{0}^{\prime \prime}$ and $K_{0}^{\prime \prime \prime}$ will not be discussed here because they are only weakly constrained (as discussed in the previous section) and are less likely than $K$, and $K_{s}^{\prime}$ to exhibit a simple dependence on material. Release behavior farther away from the Hugoniot point does depend more strongly on th " choice of $K_{s}^{\prime \prime}$ and $K_{s}^{\prime \prime \prime}$, and a further investigation of these parameters should be undertaken in the future.

The Hugoniot and release curves for two of these tests in the low-pressure region $\left(\mathrm{P}_{\mathrm{H}} \leq 15 \mathrm{GPa}\right)$ and two in the higher pressure region $\left(\mathrm{P}_{\mathrm{H}} \geq 30 \mathrm{GPa}\right)$ are plotted in Figure 3.5. These particular tests were chosen to illustrate the qualitative release behavior in tests where widely divergent moduli were observed at similar Hugoniot pressures.

Note that the pressure derivative of the axial modulus, $K_{a}^{\prime}$, is approximately 4 for the aluminum tests, while it is much larger for the MJ-2 grout (concentrated in the range $10-12$ ), and larger yet for the geological materials. Most materials under static loading show pressure derivatives of the bulk moduli (which should be comparable to the axial modulus because our deformation is one-dimensional) in the range $3-5$. This is probably due to the mixing of longitudinal and shear behaviour that makes up the observed releases. This may be understood in stress-strain space. A release for an elastic-perfectly plastic material will begin at a slope corresponding to the longitudinal sound velocity, continuing until it reaches a yield surface below the Hugoniot, then follow a path whose slope corresponds to the bulk sound velocity. Recall that the sound velocity is given by 

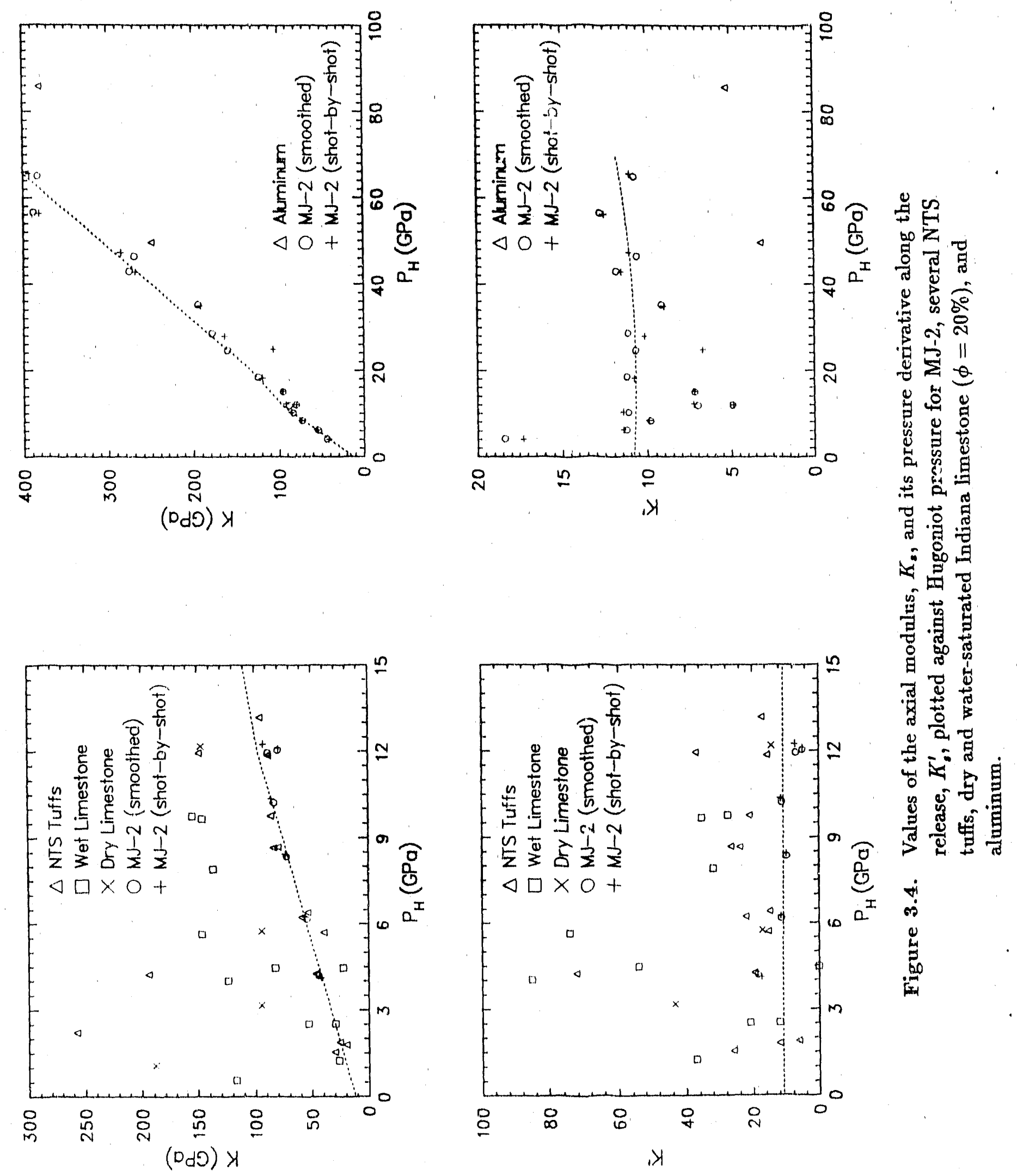


$$
C=\sqrt{\frac{K_{s}}{\rho}}, \text { where } K_{s} \equiv-\left.V\left(\frac{\partial \sigma_{x}}{\partial V}\right)\right|_{s}=\left.\rho\left(\frac{\partial \sigma_{x}}{\partial \rho}\right)\right|_{s}
$$

Such a trajectory will have a corncr. For many geological materials, we find that this corner is smeared out; release wave profiles do not have discrete arrivals of the longitudinal and bulk releases, but are dispersive. The observed axial modulus at the Hugoniot will be higher than a statically measured bulk modulus because of the finite material strength, but a large first derivative corresponds to this smeared out corner. This is well illustrated by the sample limestone release on the left side of Figure 3.5. The MJ-2 release laid alongside of it has much smaller values of both $K_{8}$ and $K_{\mathrm{a}}^{\prime}$, and is gentler and flatter. In the right hand plot of Figure 3.5, we see that the aluminum relcase (smaller $\rho / \rho_{0}$ ) lies above the Hugoniot. It reflects a nearly hydrodynamic system. The axial moduli for aluminum lie significantly below the MJ-2 moduli in Figure 3.4, and the same holds for the first derivative, $K_{s}^{\prime}$.

The behavior of the limestone and tuffs tends to reflect this corner more strongly than does the MJ-2 behavior. Larger values of $K_{s}$ and $K_{s}^{\prime}$ at a given Hugoniot condition seem to reflect a greater hysteresis, and hence either a greater Hugoniot material strength than in the grout or the existence of hysteretic phase changes in these materials. In turn, the grout show's a greater hysteresis in uniaxial strain than does the aluminum.
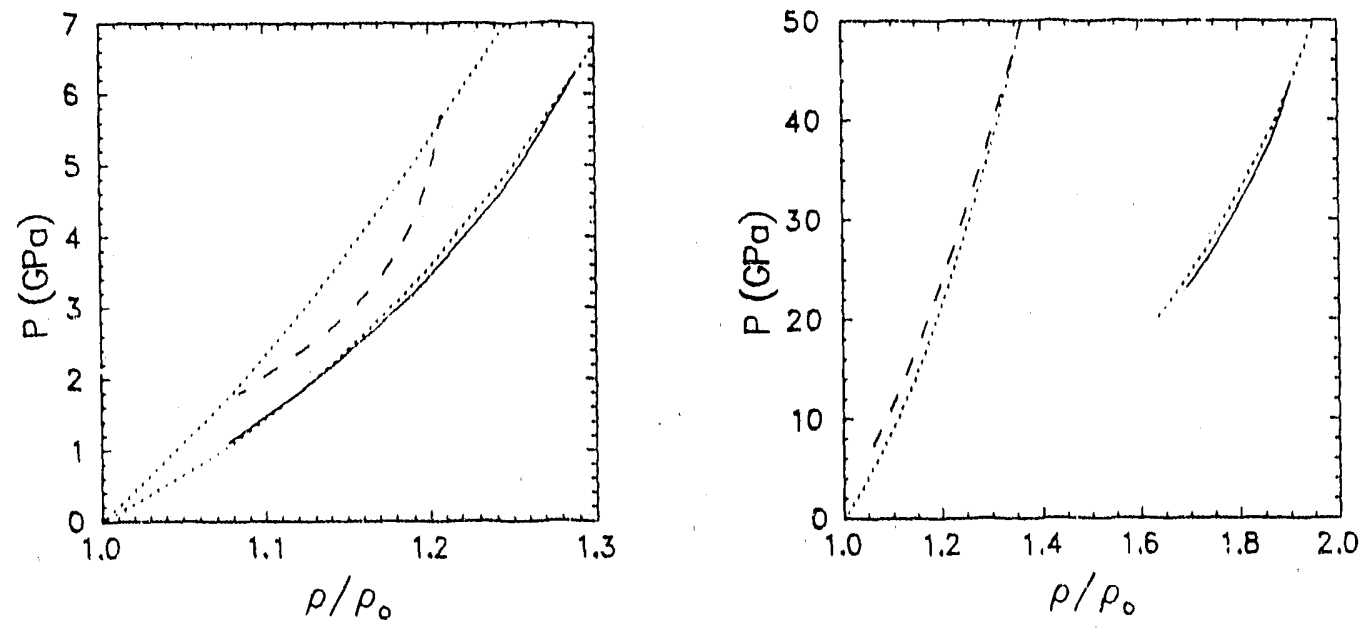

Figure 3.5. Hugoniot and release curves for two MJ-2 tests, one aluminum test and one wet limestone test, showing contrasting behavior also reflected in modulus data. Solid curves are MJ-2 releases (test DNA2 in the left plot and DNA16 in the right pot); the dashed curve in the left plot is a wet . limestone release; and that in the right plot is an aluminum release. Dotted curves are Hugoniot. Releases are drawn only as far down in pressure as they are experimenta!", constrained. 
We note in closing that the hysteresis as a whole is probably a combination of uniaxial and bulk effects. Strictly, the analytical treatments presented here deal only with stress in the direction of projectile motion, and not with pressure. The problem of distinguishing between effects due to material strength and those due to phase changes or bulk compressibility is beyond the scope of the present report. 


\section{Application of interpolatable expression of releases to a practical problem}

In one practical nuclear test configuration designed for measuring groundshock in the near- and intermediate-field range, stress and particle velocity gauges are cast into a large grout hemisphere centered on the working point (site of nuclear device). Pressures achieved in the grout at the gauge positions are of the same order as the pressures achieved in the gun tests mentioned earlier.

Let us briefly state a sample problem. If a shock wave propagating through the grout impinges on a detector (or other equipment) with different shock impedance from that of the grout, a shock or release will be reflected back into the grout. We may make a particle velocity measurement of this reshocked or partially released state, and try to determine the pressure from this measurement. How much error is introduced by ignoring the shock-release or double-shock history of the sample and calculating the pressure based on the single-shock Hugoniot?

For the reshock case (where the shock impinges on aluminum or magnesium, for example), we do not have new experimental data and must rely on assumed equations of state such as a Mie-Grüneison model.

For the release case (a shock impinging on PMMA), we may either use a MieGrüneison model or attempt to apply our experimental results. The technique discussed in Chapter 2 provides a way of applying the experimental results to this problem; ie. it allows us to determine from the experimental release data how the grout will release from the Hugoniot pressures dictated by the problem.

The problem is described schematically in Figure 4.1. A shock wave of (known) pressure $P_{1}$ and particle velocity $U_{P 1}$ travels through $\mathrm{MJ}-2$ grout, compressing it to specific volume $V_{1}$. This shock wave enters a mass of aluminum, magnesium or polymethyl methacrylate (PMMA), and a reshock (aluminum or magnesium) or release wave (PMMA) travels back into the MJ-2. Material behind this second wave is at pressure $P_{2}$, particle velocity $U_{P_{2}}$ and specific volume $V_{2}$. We wish to compute $P_{2}, U_{P_{2}}$ and $V_{2}$ as accurately as possible, then compare this result with:

1. The pressure we would calculate if we measure the final particle velocity $U_{P_{2}}$ and assume that reshocks and releases for MJ-2 follow the principal Hugoniot. This is a likely experimental situation. The pressure is calculated as:

$$
P_{P H}=\rho_{0}\left(C_{0}+S U_{P(\mathrm{eff})}\right) \cdot U_{P(\mathrm{eff})},
$$



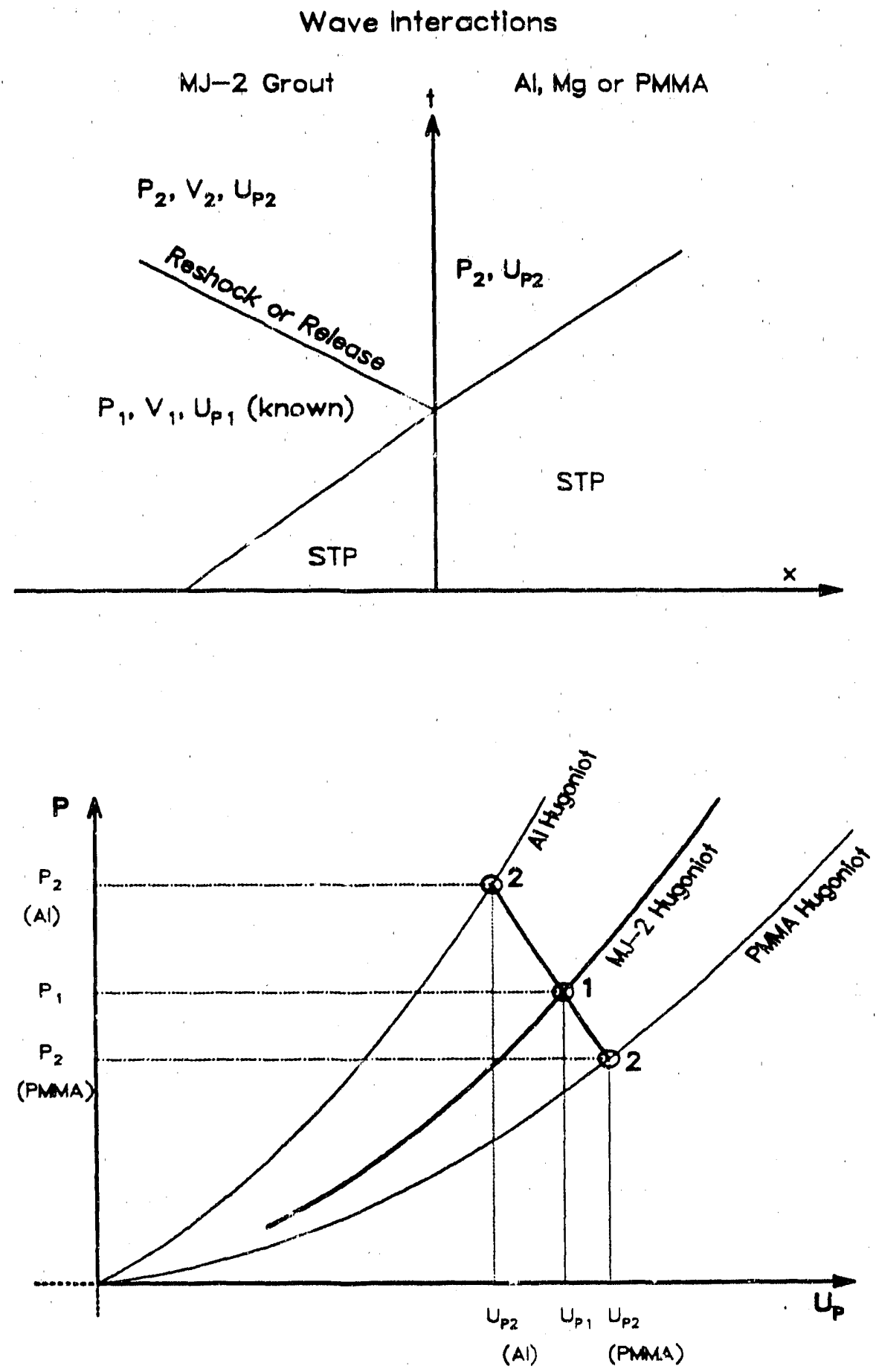

Figure 4.1. Schematic of the gauge problem 
where:

$U_{P(\mathrm{eff})} \equiv 2 U_{P 1}-U_{P 2}$.

is a sum of changes in particle velocity ("PH" stands for "Principal Hugoniot").

2. The final specific volume we would calculate if we nueasure the final pressure $P_{2}$ and mais the same assumption as above. This is calculated as:

$V_{P H}=V_{0} \cdot\left(U_{S(P H)}-U_{P(P H)}\right) / U_{S(P H)}$

with

$U_{S(P H)}=C_{0}+s U_{P(P H)}$

and

$U_{P(P H)}=\left[-\rho_{0} C_{0}+\sqrt{\left(\rho_{0} C_{0}\right)^{2}+4 P_{2} \rho_{0} s}\right] /\left(2 \rho_{0} s\right)$.

3. The pressure we would calculate if we measure the final volume $V_{2}$ and make the same assumption as above. This is:

$P_{P_{H}}=\left(\rho_{0} C_{0}^{2} \eta\right) /(1-s \eta)^{2}$,

where, as is customary,

$\eta=1-V_{2} / V_{0}$

The basic procedure for this is indicated in Fig. 4.1, and is best seen in $\mathrm{P} / \mathrm{U}_{\mathrm{P}}$ space. We begin by computing the centering point on the MJ-2 principal Hugoniot (pressure $\mathrm{P}_{1}$ ) for a release or reshock.

If we use a Mie-Grüneison equation of state (for reshock or release), we next calculate a trajectory in pressure-volume space based on procedures described iy McQueen et al. (1970):

$$
P \equiv P_{2}\left(V_{2}, V_{1}\right)=\frac{P_{H}-(\rho \gamma)_{2}\left[\left(P_{H}-P_{1}\right)\left(V_{0}-V_{2}\right) / 2\right]}{1-(\rho \gamma)_{2}\left(V_{1}-V_{2}\right) / 2}
$$

(where the subscript 1 refers to the centering point of the reshock or release, subscript 2 refers to any state along the reshuck or release, and $\mathrm{P}_{\mathrm{H}}$ represents the single-shock Hugoniot pressure at specific volume $V_{2}$ ). This may be converted to pressure-particle velocity space using the relation $U_{P_{2}}=U_{P_{1}}-\sqrt{\left(V_{2}-V_{1}\right)\left(P_{2}-P_{1}\right)}$ (where we have assumed that the reshock or release is due to a steady-profile wave travelling in the opposite direction from the first shock). The intersection between this trajectory and the principal Hugoniot of the other material is then the reshocked or partially released state of the grout. 
If, however, we use a release trajectory based on our experimental data, we compute a pressure-volume release trajectory based on fits to the release axial modulus $K_{s}\left(P, P_{H}\right)$ and its pressure derivatives, as described above. This is then transformed to pressureparticle velocity space in the same way as before.

We have done these calculations for initial shocks of amplitudes of 5-100 GPa $(5,10$, then at $10 \mathrm{GPa}$ intervals) impinging on aluminum, magnesium and PMMA. For cases which generated releases (those with PMMA), we have made these calculations assuming a Mie-Grüneison EOS and repeated them using instead the modulus information generalized from our experiments. All of these data are summarized in Table 4.1 ( $\mathrm{Al}$ and $\mathrm{Mg}$ ) and Table 4.2 (PMMA). The errors caused by assuming that releases and reshocks follow the principal Hugoniot (as detailed for the three cases above) are graphically shown as percentages in Figs. 4.2 and 4.3. Positive percentages mean that the approximate procedure will overestimate the quantity.

Figure 4.4 shows the trajectories actually calculated, in $\mathrm{P} / \mathrm{U}_{\mathrm{P}}$ space, and gives some physical intuition for how the errors originate. For example, the vertical arrows show the errors in calculated pressure obtained from a particle velocity measurement and the assumption that the release trajectory follows the principal Hugoniot (or a translated mirror image thereof). One needs to be aware that anything beyond $70 \mathrm{GPa}$ in the grout is an extrapolation and should be used cautiously. 


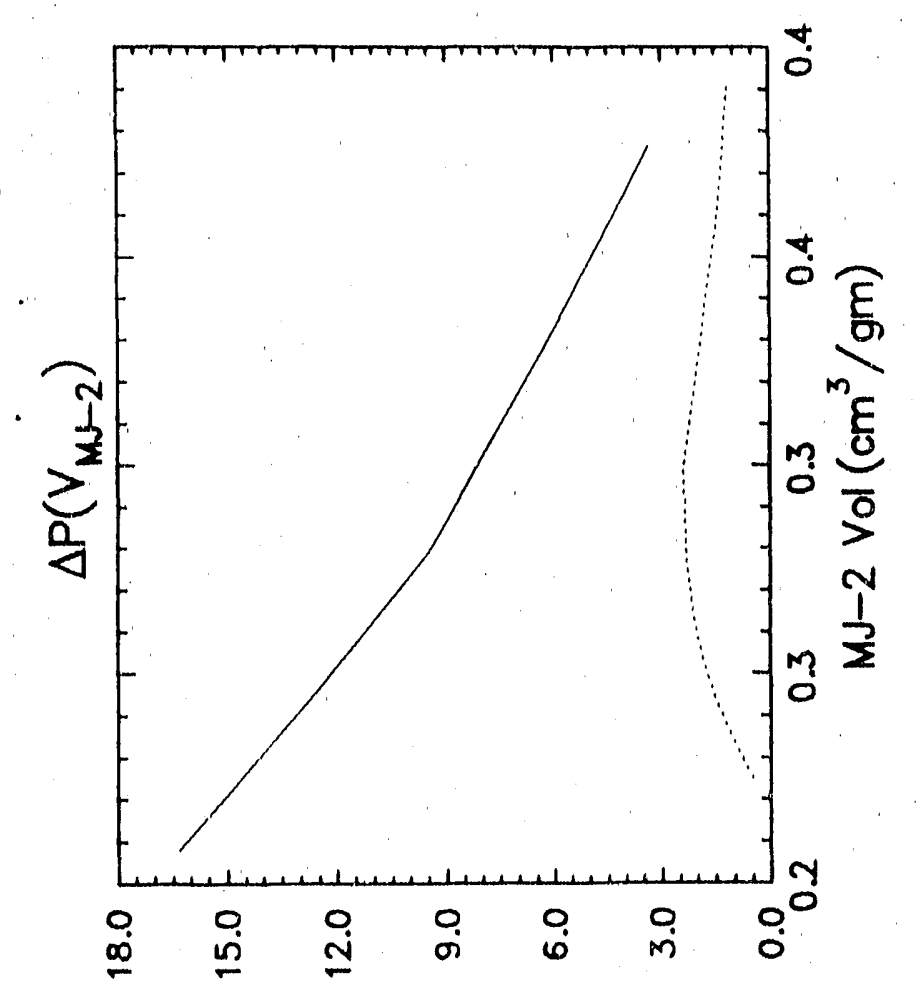

(\%) o.nssedd U

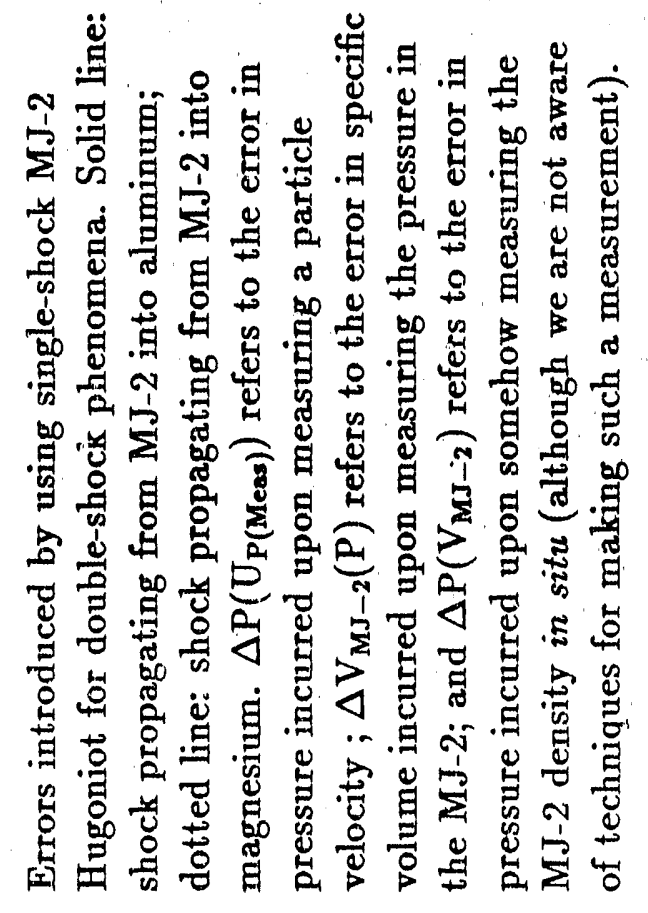

苟

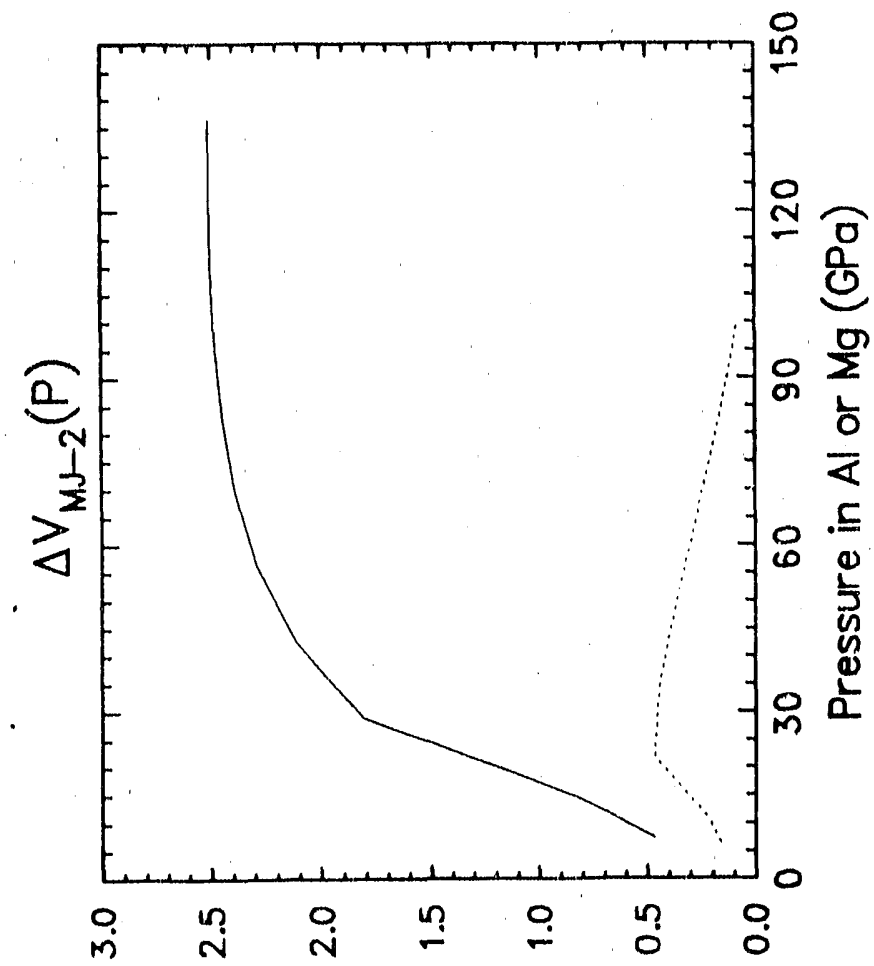

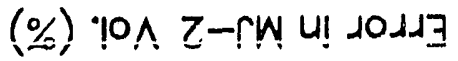

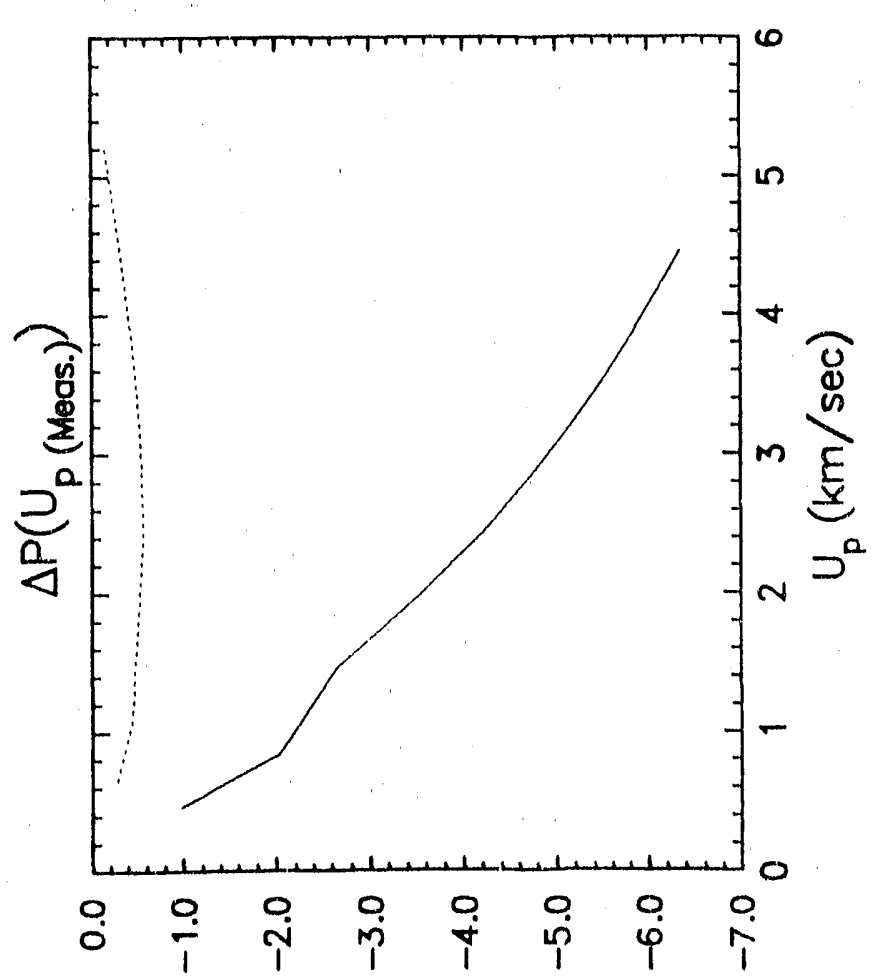

(\%) odinssodd ui jodiz 


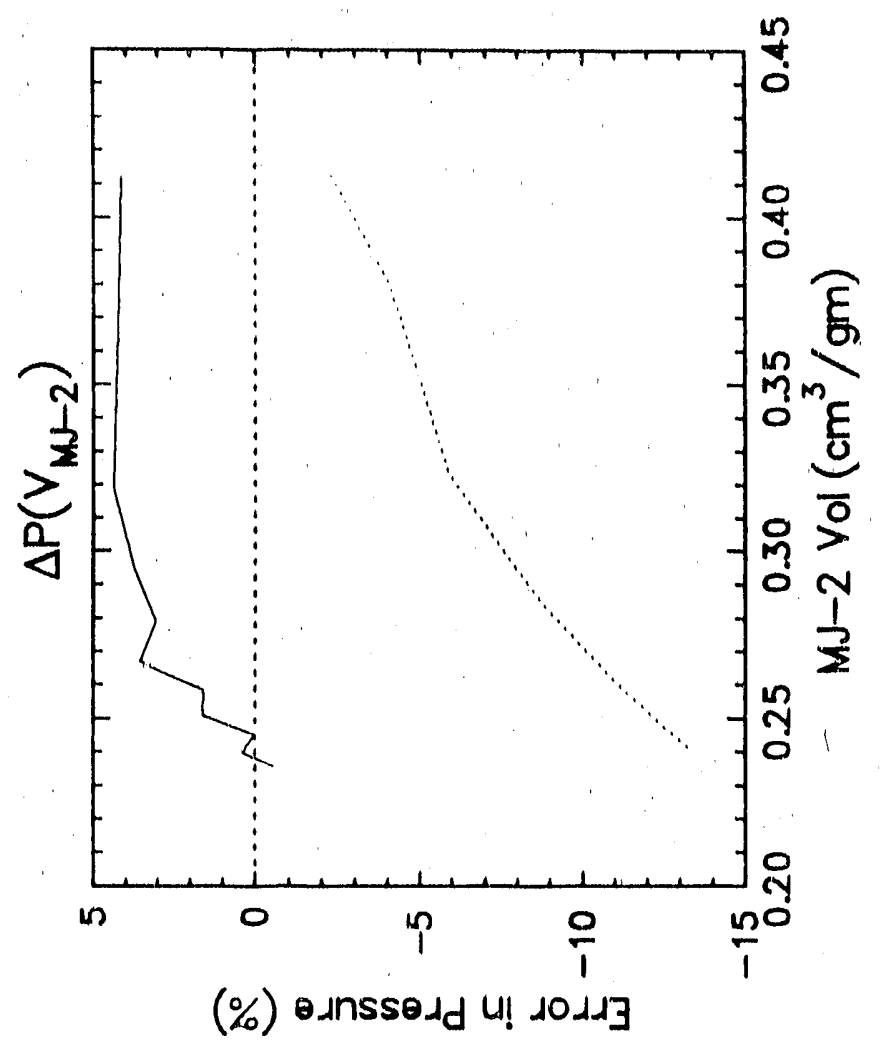

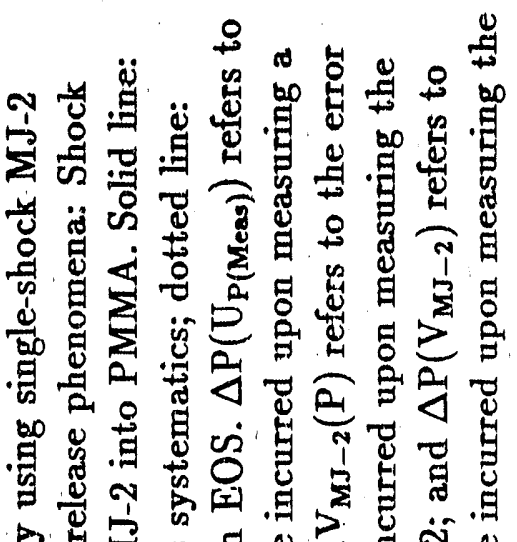

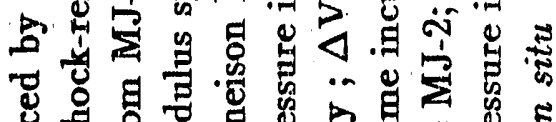

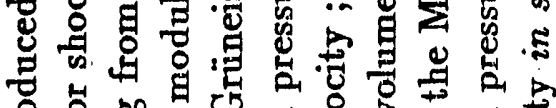

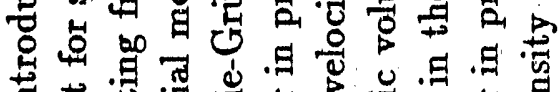

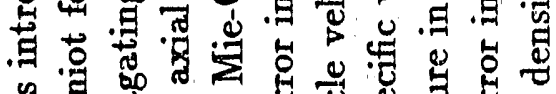

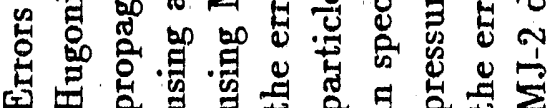

में

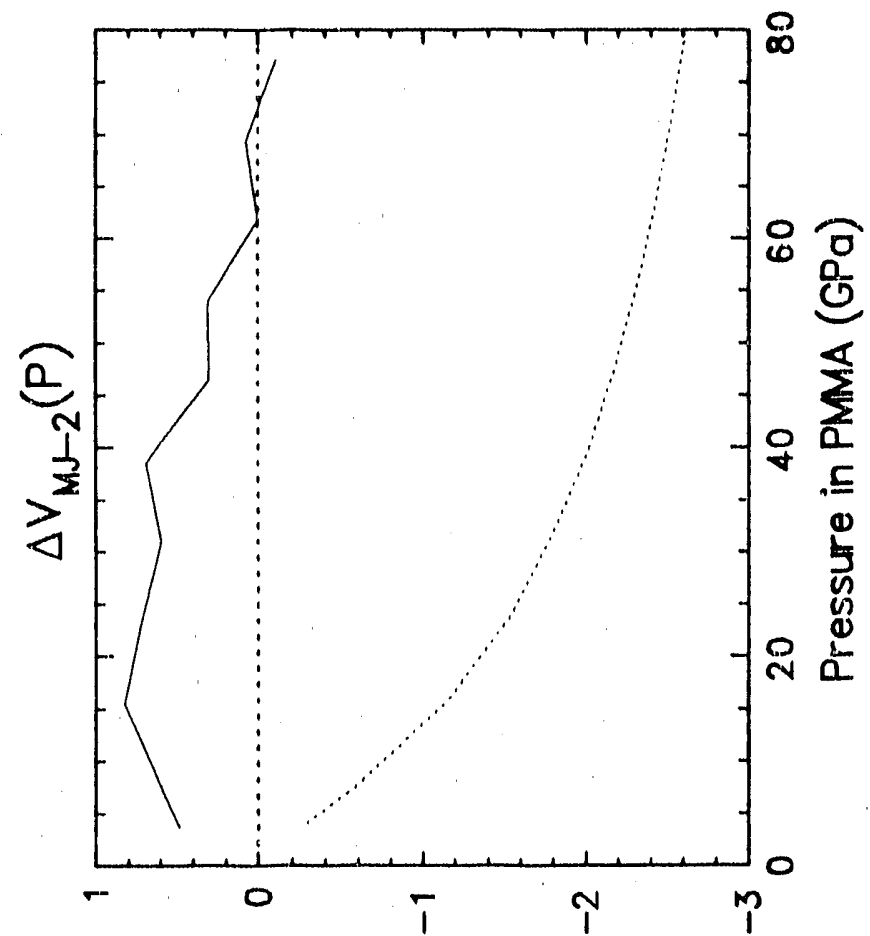

(\%) $" 0 \wedge \tau-\Gamma W ~ 4 !$

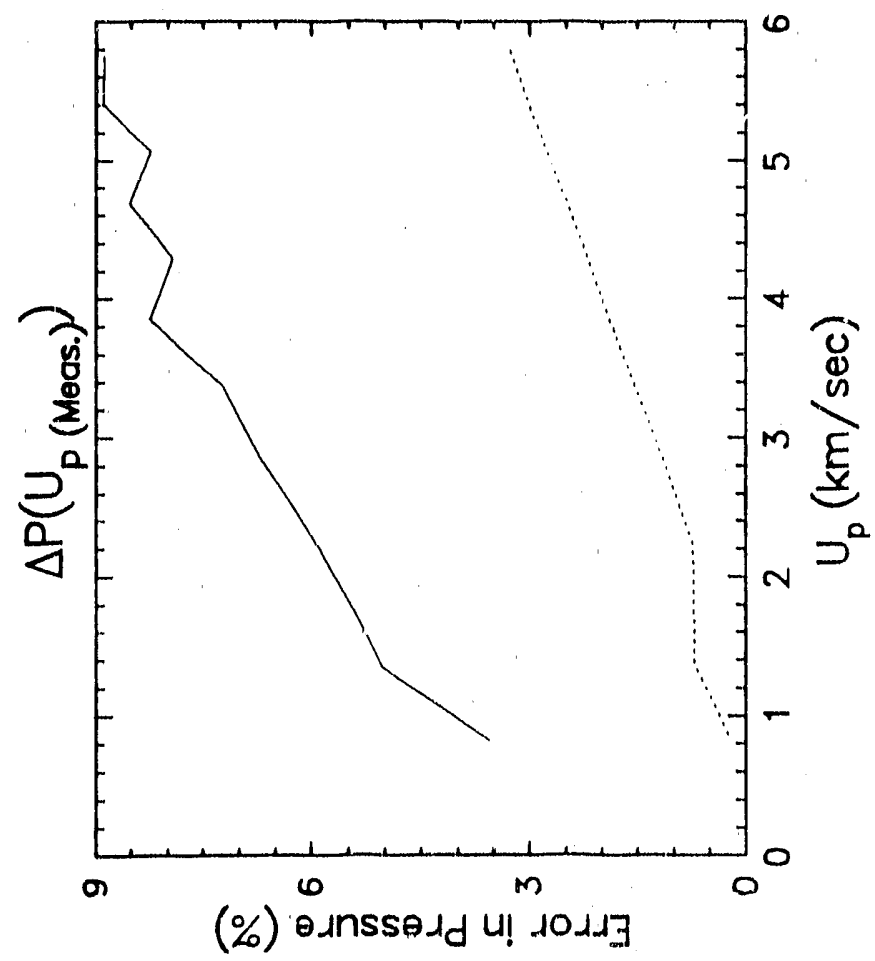


Table 4.1. Summary of analysis of system with shock propagating from MJ-2 into aluminum or magnesium. EOS constants for MJ-2 taken as: $\mathrm{C}_{0}=2.4, \mathrm{~S}$ $=1.6\left(\mathrm{P}_{\mathrm{H}} \leq 20 \mathrm{GPa}\right) ; \mathrm{C}_{0}=2.4, \mathrm{~S}=1.36\left(\mathrm{P}_{\mathrm{H}}>20 \mathrm{GPa}\right) ; \rho \gamma=3.00$. Subscripts: "1" refers to state in MJ-2 after passage of first shock; "2" refers to state in MJ-2 after passage of second shock; and " $\mathrm{P}_{\mathrm{H}}$ " refers to assumption that reshock coincides with principal Hugoniot or its translated mirror image in calculating the listed value from the (measured) arguement $P_{2}, V_{2}$ or $U_{P_{2} 2}$. Percentage errors refer to the deviation of $\mathrm{P}_{\mathrm{P}_{H}}$ or $\mathrm{V}_{\mathrm{P}_{H}}$ from the "correctly" (multi-shock) calculated quantity $\mathrm{V}_{2}$ or $\mathrm{P}_{2}$.

\begin{tabular}{|c|c|c|c|c|c|c|c|c|c|c|}
\hline \multicolumn{11}{|c|}{ (Shock propagating into aluminum; $C_{0}=5.37, S=1.34, \rho_{0}=2.6978$ ) } \\
\hline $\begin{array}{c}P_{1} \\
\left(G P_{a}\right)\end{array}$ & $\begin{array}{c}U_{P, 1} \\
(\mathrm{~km} / \mathrm{sec})\end{array}$ & $\begin{array}{c}\mathrm{P}_{2} \\
(\mathrm{GPa})\end{array}$ & $\begin{array}{c}V_{2} \\
\left(m^{3} / M_{13}\right)\end{array}$ & $\begin{array}{c}\mathrm{U}_{\mathrm{P},{ }^{2}} \\
(\mathrm{~km} / \mathrm{sec})\end{array}$ & $\begin{array}{l}V_{P_{H}}\left(P_{2}\right) \\
\left(m^{j} / M_{g}\right)\end{array}$ & $\begin{array}{c}\% \\
\text { Error } \\
\end{array}$ & $\begin{array}{c}\mathrm{PP}_{\mathrm{P}_{1}}\left(\mathrm{~V}_{2}\right) \\
(\mathrm{GPa}) \\
\end{array}$ & $\begin{array}{c}\% \\
\text { Error }\end{array}$ & $\begin{array}{c}P_{P_{1}(}\left(U_{\left.P_{12}\right)}\right) \\
\left(G P_{a}\right)\end{array}$ & $\begin{array}{c}\% \\
\text { Eirror }\end{array}$ \\
\hline 5 & 0.708 & 7.524 & 0.3765 & 0.465 & 0.3783 & 0.47 & 7.775 & 3.34 & 7.450 & -0.98 \\
\hline 10 & 1.170 & 14.712 & 0.3398 & 0.840 & 0.3426 & 0.82 & 15.528 & 5.55 & 14.414 & -2.03 \\
\hline 20 & 1.969 & 29.160 & 0.2791 & 1.472 & 0.2842 & 1.81 & 31.933 & 9.51 & 28.385 & -2.66 \\
\hline 30 & 2.654 & 43.022 & 0.2578 & 1.986 & 0.2633 & 2.12 & 47.839 & 11.43 & 41.500 & -3.54 \\
\hline 40 & 3.053 & 56.670 & 0.2440 & 2.434 & 0.2496 & 2.28 & 63.888 & 12.74 & $E 4.291$ & -4.20 \\
\hline 50 & 3.495 & 70.175 & 0.2342 & 2.836 & 0.2398 & 2.39 & 79.788 & 13.70 & 66.862 & -4.72 \\
\hline 60 & 3.896 & 83.574 & 0.2287 & 3.205 & 0.2322 & 2.45 & 85.648 & 14.45 & 78.270 & -5.15 \\
\hline 70 & 4.267 & 96.893 & 0.2207 & 3.548 & 0.2262 & 2.48 & 111.474 & 15.05 & 91.652 & -5.51 \\
\hline 80 & 4.612 & 110.148 & 0.2158 & 3.869 & 0.2211 & 2.50 & 127.273 & 15.55 & 103.732 & -5.83 \\
\hline 90 & 4.937 & 123.350 & 0.2116 & 4.172 & 0.2169 & 2.51 & 143.048 & 15.97 & 115.827 & -6.10 \\
\hline 100 & 5.245 & 136.508 & 0.2080 & 4.460 & 0.2132 & 2.51 & 158.804 & 16.33 & 127.850 & -6.34 \\
\hline \multicolumn{11}{|c|}{ (Shock propagating into magnesiurn; $C_{0}=4.492, S=1.263, \rho_{0}=1.74$ ) } \\
\hline $\begin{array}{c}P_{1} \\
\left(G P_{a}\right)\end{array}$ & $\begin{array}{c}U_{P_{11}} \\
(\mathrm{~km} / \mathrm{sec})\end{array}$ & $\begin{array}{c}\mathrm{P}_{2} \\
(\mathrm{GPa})\end{array}$ & $\begin{array}{c}V_{2} \\
\left(\mathrm{~m}^{3} / \mathrm{Mg}\right)\end{array}$ & $\begin{array}{c}U_{\mathrm{P}, 2} \\
(\mathrm{~km} / \mathrm{sec})\end{array}$ & $\begin{array}{l}\mathrm{V}_{\mathrm{P}_{H}}\left(\mathrm{P}_{2}\right) \\
\left(\mathrm{m}^{3} / \mathrm{Mg}_{\mathrm{g}}\right) \\
\end{array}$ & $\begin{array}{c}\% \\
\text { Error }\end{array}$ & $\begin{array}{c}\mathrm{P}_{\mathrm{P}_{\mathbf{H}}}\left(\mathrm{V}_{2}\right) \\
(\mathrm{GP})\end{array}$ & $\begin{array}{c}\% \\
\text { Error }\end{array}$ & $\begin{array}{c}\mathrm{P}_{\mathrm{P}_{H}}\left(\mathrm{U}_{\mathrm{P}, 2}\right) \\
(\mathrm{GPa})\end{array}$ & $\begin{array}{c}\% \\
\text { Error }\end{array}$ \\
\hline 5 & 0.708 & 5.778 & 0.3917 & 0.628 & 0.3923 & 0.15 & 5.845 & $\overline{1.16}$ & 5.762 & -0.27 \\
\hline 10 & 1.170 & 11.094 & 0.3567 & 1.087 & 0.3575 & 0.22 & 11.259 & 1.40 & 11.045 & -0.44 \\
\hline 20 & 1.969 & 21.992 & 0.2991 & 1.851 & 0.3005 & 0.47 & 22.519 & 2.39 & 21.877 & -0.62 \\
\hline 30 & 2.554 & 32.250 & 0.2773 & 2.445 & 0.2786 & 0.45 & 32.996 & 2.31 & 32.066 & -0.57 \\
\hline 40 & 3.053 & 42.318 & 0.2630 & 2.957 & 0.2641 & 0.40 & 43.204 & 2.09 & 42.083 & -0.56 \\
\hline 50 & 3.495 & 52.257 & 0.2527 & 3.412 & 0.2535 & 0.35 & 53.209 & 1.82 & 51.990 & -0.51 \\
\hline 60 & 3.896 & 62.098 & 0.2447 & 3.827 & 0.2454 & 0.28 & 63.053 & 1.54 & 61.820 & -0.45 \\
\hline 70 & 4.267 & 71.864 & 0.2382 & 4.210 & 0.2388 & 0.23 & 72.764 & 1.25 & 71.693 & -0.38 \\
\hline 30 & 4.612 & 81.567 & 0.2329 & 4.568 & 0.2333 & 0.18 & 82.362 & 0.97 & 81.321 & -0.30 \\
\hline 80 & 4.937 & 91.217 & 0.2283 & 4.905 & 0.2286 & 0.13 & 91.861 & 0.71 & 81.014 & -0.22 \\
\hline 100 & 5.245 & 100.823 & 0.2244 & 5.225 & 0.2246 & 0.08 & 101.274 & 0.45 & 100.678 & -0.14 \\
\hline
\end{tabular}


Table 4.2. Summary of analysis of system with shock propagating from MJ-2 into PMMA. EOS constants for MJ-2 taken as in Table 4.1; for PMMA: $\mathrm{C}_{0}=2.59, \mathrm{~S}=1.52$ and $\rho_{0}=1.185$. Subscripts: "1" refers to state in MJ-2 after passage of first shock; "2" refers to state in MJ-2 after passage of release; and " $\mathrm{P}_{\mathrm{H}}$ " refers to assumption that release coincides with principal Hugoniot or its translated mirror image in calculating the listed value from the (measured) arguement $P_{2}, V_{2}$ or $U_{P, 2}$. Percentage errors refer to the deviation of $\mathrm{P}_{\mathrm{P}_{\mathrm{H}}}$ or $\mathrm{V}_{\mathrm{P}_{\mathrm{H}}}$ from the "correctly" (shock-release) calculated quantity $\mathrm{V}_{2}$ or $\mathrm{P}_{2}$.

\begin{tabular}{|c|c|c|c|c|c|c|c|c|c|c|}
\hline \multicolumn{11}{|c|}{ (Assuming Mie. Grüneison EOS for MJ-2 upon release) } \\
\hline $\begin{array}{c}\mathrm{P}_{1} \\
(\mathrm{GPa})\end{array}$ & $\begin{array}{c}U_{p, 1} \\
(\mathrm{kmn} / \mathrm{sec})\end{array}$ & $\begin{array}{c}\mathrm{P}_{2} \\
(\mathrm{GPa})\end{array}$ & $\begin{array}{c}\mathrm{V}_{2} \\
\left(\mathrm{~m}^{\mathrm{g}} / \mathrm{Mg}_{\mathrm{g}}\right)\end{array}$ & $\begin{array}{c}\mathrm{U}_{\mathrm{P}, 2} \\
(\mathrm{~km} / \mathrm{sec})\end{array}$ & $\begin{array}{l}V_{\mathrm{P}_{H}}\left(\mathrm{P}_{2}\right) \\
\left(\mathrm{m}^{3} / \mathrm{Mg}_{\mathrm{g}}\right) \\
\end{array}$ & $\begin{array}{c}\% \\
\text { Error }\end{array}$ & $\begin{array}{c}P_{P_{H}}\left(V_{2}\right) \\
\left(G P_{B}\right) \\
\end{array}$ & $\begin{array}{l}\% \\
\text { Error }\end{array}$ & $\begin{array}{c}\operatorname{PP}_{P_{H}}\left(U_{P_{1}, 2}\right) \\
\left(G P_{a}\right)\end{array}$ & $\begin{array}{c}\% \\
\text { Ertor }\end{array}$ \\
\hline 5 & 0.708 & 3.834 & 0.4143 & 0.838 & 0.4132 & -0.26 & 3.750 & -2.21 & 3.843 & 0.21 \\
\hline 10 & 1.170 & 7.600 & 0.3800 & 1.372 & 0.3777 & -0.88 & 7.291 & $-4,06$ & 7.653 & 0.70 \\
\hline 20 & 1.969 & 15.900 & 0.3238 & 2.239 & 0.3201 & -1.16 & 14.963 & -5.89 & 16.015 & 0.73 \\
\hline 30 & 2.554 & 23.838 & 0.3003 & 2.884 & 0,2957 & -1.53 & 22.045 & -7.52 & 24.110 & 1.18 \\
\hline 40 & 3.053 & 31.758 & 0.2845 & 3.433 & 0.2794 & -1.80 & 28.967 & -8.79 & $32.25 \theta$ & 1.58 \\
\hline 50 & 3.495 & 39.663 & 0.2730 & 3.817 & 0.2675 & -2.01 & 35.764 & -9.83 & 40.428 & 1.83 \\
\hline 60 & 3.896 & 47.552 & 0.2638 & 4.356 & 0.2582 & -2.18 & 42.458 & -10.71 & 48.622 & 2.25 \\
\hline 70 & 4.267 & 55.428 & 0.2566 & 4.760 & 0.2507 & -2.31 & 49.065 & $-11,48$ & 56.837 & 2.54 \\
\hline 80 & 4.612 & 63.292 & 0.2506 & 5.137 & 0.2445 & -2.43 & 55.587 & .12 .16 & 65.071 & 2.81 \\
\hline 90 & 4.937 & 71.144 & 0.2454 & 5.190 & 0.2392 & -2.52 & 62.062 & -12.77 & 73.322 & 3.06 \\
\hline 100 & 5.245 & 78.987 & 0.2409 & 5.825 & 0.2346 & -2.60 & 68.467 & -13.32 & 81.588 & 3.29 \\
\hline \multicolumn{11}{|c|}{ (Using interpolated axial modulus doscription of release) } \\
\hline $\begin{array}{c}P_{1} \\
\left(G_{a}\right)\end{array}$ & $\begin{array}{c}U_{P, 1} \\
(\mathrm{~km} / \mathrm{sec})\end{array}$ & $\begin{array}{c}P_{2} \\
\left(G P_{0}\right)\end{array}$ & $\begin{array}{c}\mathrm{V}_{2} \\
\left(\mathrm{~m}^{3} / \mathrm{Mg}_{\mathrm{g}}\right)\end{array}$ & $\begin{array}{c}\mathrm{U}_{\mathrm{P}, \mathrm{I}} \\
(\mathrm{km} / \mathrm{sec})\end{array}$ & $\begin{array}{l}V_{P_{j^{4}}}\left(P_{2}\right) \\
\left(m^{3} / M_{G}\right)\end{array}$ & $\begin{array}{c}\% \\
\text { Error }\end{array}$ & $\begin{array}{c}\mathrm{P}_{\mathrm{P}_{H}}\left(\mathrm{~V}_{2}\right) \\
(\mathrm{GPB})\end{array}$ & $\begin{array}{c}\% \\
\text { Error }\end{array}$ & $\begin{array}{c}\mathrm{P}_{\mathrm{P}_{n}}\left(\mathrm{U}_{\mathrm{P}, 2}\right) \\
(\mathrm{GPa})\end{array}$ & $\begin{array}{c}\% \\
\text { Error }\end{array}$ \\
\hline 5 & 0.708 & 3.782 & 0.4118 & $0.82 \theta$ & 0.4139 & 0.48 & 3.938 & 4.13 & 3.916 & 3.66 \\
\hline 10 & 1.170 & 7.465 & $0.37 A 5$ & 1.355 & 0.3787 & 0.59 & 7.781 & 4.23 & 7.840 & 5.03 \\
\hline 20 & 1.869 & 15.549 & 0.3188 & 2.207 & 0.3215 & 0.83 & 16.227 & 4,36 & 16.463 & 5.88 \\
\hline 30 & 2.554 & 23.277 & 0.2950 & 2.842 & 0.2871 & 0.72 & 24.145 & 3.73 & 24.832 & 6.68 \\
\hline 40 & 3.053 & 30.991 & 0.2791 & 3.383 & 0.2808 & 0.60 & 31.938 & 3.06 & 33.234 & 7.24 \\
\hline 50 & 3.495 & 38.597 & 0.2671 & 3.855 & 0.2689 & 0.69 & 39.974 & 3.57 & 41.782 & 8.25 \\
\hline 60 & 3.896 & 46.403 & 0.2586 & 4.295 & 0.2594 & 0.31 & 47.151 & 1.61 & 50.080 & 7.92 \\
\hline 70 & 4.267 & 54.021 & 0.2511 & 4.690 & 0.2519 & 0.31 & 54.814 & 1.65 & 58.623 & 8.52 \\
\hline 80 & 4.612 & 61.831 & 0.2456 & 5.069 & 0.2456 & 0.00 & 61.831 & 0.00 & 06.825 & 8.24 \\
\hline 90 & 4.937 & 69.385 & 0.2401 & 5.413 & 0.2403 & 0.07 & 69.662 & 0.40 & $75.557^{\circ}$ & 8.80 \\
\hline 100 & 5.245 & 77.117 & 0.2350 & 5.747 & 0.2357 & -0.10 & 76.609 & $-0,54$ & 83.965 & 8.88 \\
\hline
\end{tabular}




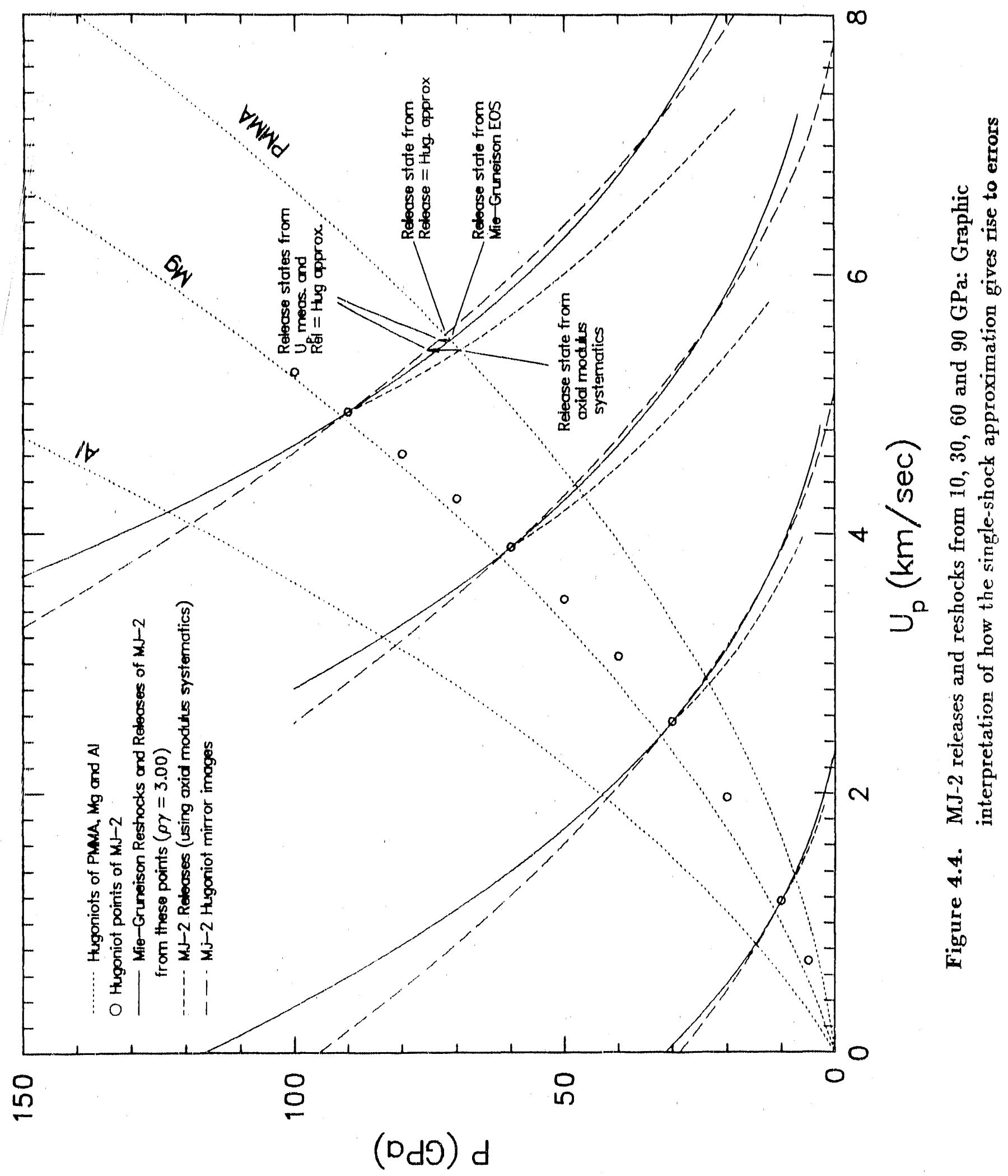




\section{Acknowledgements}

I am pleased to thank Al Chabai for interesting me in the application discussed in Chapter 4, and Dennis Grady for encouraging me to see what information about moduli could be gleaned from the MJ.2 results we had already obtained. 


\section{References}

1. Furnish, M. D., Measuring the dynamic compression and release behavior of the Paintbrush and Tunnelbed (NTS) Tuffs over the range 1-13 GPa, Sandia National Laboratories Report SAND90-00000, 1990a.

2. Furnish, M. D., Dynamic compression and release experiments on Indiana lime. stone, in Shock Waves in Condensed Matter, S. Schmitt, J. N. Johnson and L. W. Davison (eds), North-Holland, pp. 625-628, 1990 b.

3. Furnish, M. D., Dynamic compression and release experiments on Indiana limestone, Sandia National Laboratories Report, in preparation, 1990c.

4. Grady, D. E. and M. D. Furnish, Shock and release-wave properties of MJ-2 grout, Sandia National Laboratories Rept. SAND88-1642, December, 1988.

5. Hermann, W., Constitutive equation for the dynamic compaction of ductile porous materials, J. Appl. Phys., 40, 2490, 1968.

6. Jeanloz, R. an 1 T. J. Ahrens, Release adiabat measurements on minerals: the effect of viscosity, J. Geophys. Res., 84,7545-7548, 1979.

$\because$ Kipp, M. E. and R. J. Lawrence, WONDY V - A one-dimensional finite-difference wave propagation code, Sandia National Laboratories Rept. SAND81-0930, June 1982.

8. Larson, D. B, and G. D. Anderson, Plane shock wave studies of porous geologic media, J. Geophys. Res., 84, 4592-4600, 1979.

9. R. G. McQueen, S. P. Marsh, J. W. Taylor, J. N. Fritz and W. J. Carter, The equation of state of solids from shock wave studies, pp. 294-417 in R. Kinslow (ed.), High Velocity Impact Phenomena, Academic Press, 1970

10. Murri, W. J., D. E. Grady and K. D. Mahrer, Equation of state of rocks, SRI Report, SRI Project PYU.1883, 1975.

11. Peterson, C. F., W.J. Murri and M. Cowperthwaite, Hugoniot and release-adiabat measurements for selected geologic materials, J. Geophys. Res., 75, 2063-2072, 1970.

12. Rosenberg, J. T., T. J. Ahrens and C. F. Petersen, Dynamic properties of rocks, SRI Report, SRI Project PGU-6273, 1968. 

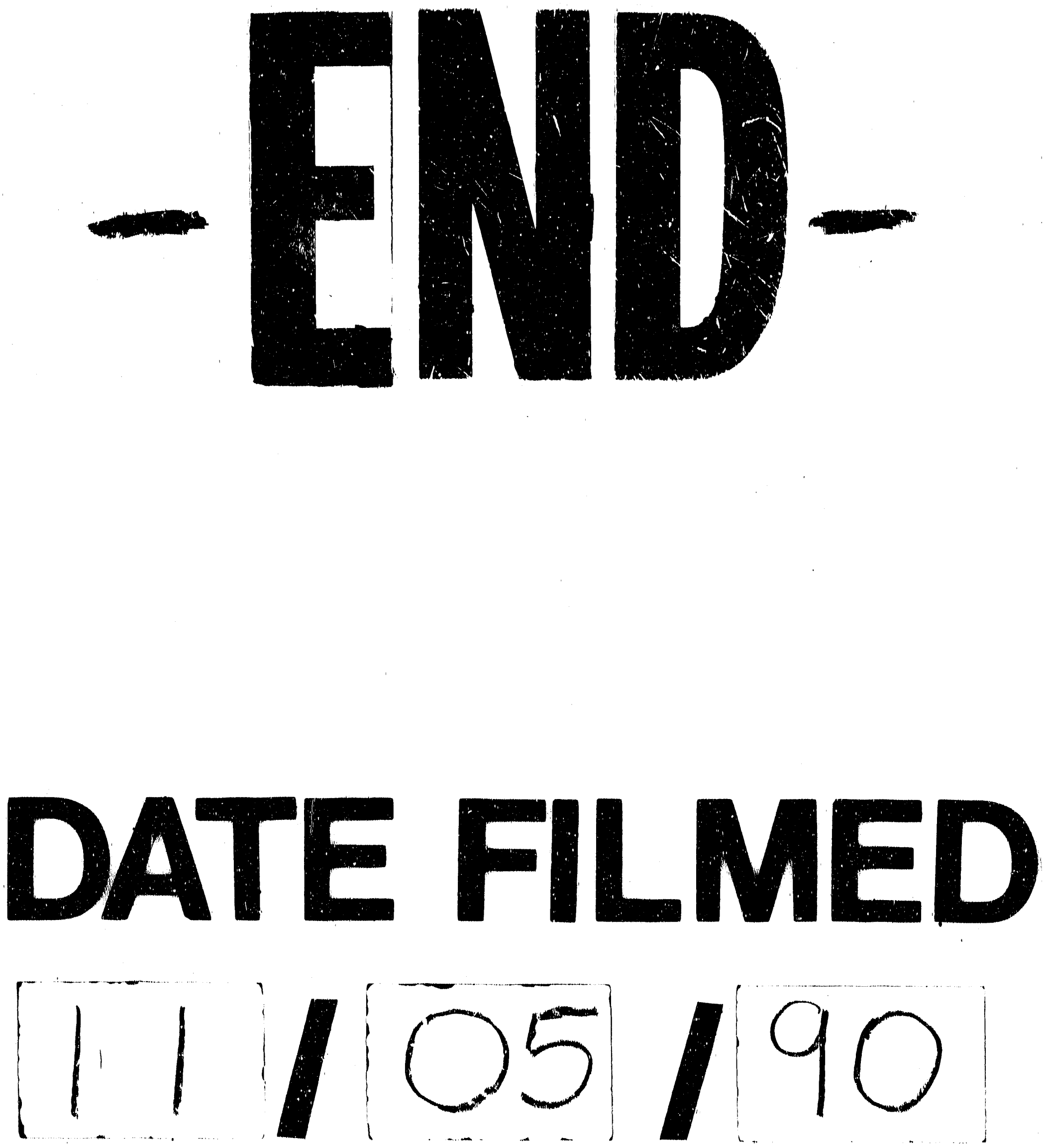
